\title{
Quantized Hall Conductance of a Single Atomic Wire: A Proposal Based on Synthetic Dimensions
}

\author{
G. Salerno®, ${ }^{1, *}$ H. M. Price, ${ }^{2}$ M. Lebrat, ${ }^{3}$ S. Häusler, ${ }^{3}$ T. Esslinger, ${ }^{3}$ L. Corman, ${ }^{3}$ J.-P. Brantut, ${ }^{4}$ and N. Goldman ${ }^{1, \dagger}$ \\ ${ }^{1}$ Center for Nonlinear Phenomena and Complex Systems, Université Libre de Bruxelles, \\ CP 231, Campus Plaine, B-1050 Brussels, Belgium \\ ${ }^{2}$ School of Physics and Astronomy, University of Birmingham, \\ Edgbaston, Birmingham B15 2TT, United Kingdom \\ ${ }^{3}$ Institute for Quantum Electronics, ETH Zurich, 8093 Zurich, Switzerland \\ ${ }^{4}$ Institute of Physics, EPFL, 1015 Lausanne, Switzerland
}

(Received 12 November 2018; revised manuscript received 25 June 2019; published 1 October 2019)

\begin{abstract}
We propose a method by which the quantization of the Hall conductance can be directly measured in the transport of a one-dimensional atomic gas. Our approach builds on two main ingredients: (1) a constriction optical potential, which generates a mesoscopic channel connected to two reservoirs, and (2) a timeperiodic modulation of the channel specifically designed to generate motion along an additional synthetic dimension. This fictitious dimension is spanned by the harmonic oscillator modes associated with the tightly confined channel, and hence, the corresponding "lattice sites" are intimately related to the energy of the system. We analyze the quantum-transport properties of this hybrid two-dimensional system, highlighting the appealing features offered by the synthetic dimension. In particular, we demonstrate how the energetic nature of the synthetic dimension combined with the quasienergy spectrum of the periodically driven channel allows for the direct and unambiguous observation of the quantized Hall effect in a two-reservoir geometry. Our work illustrates how topological properties of matter can be accessed in a minimal one-dimensional setup, with direct and practical experimental consequences.
\end{abstract}

DOI: 10.1103/PhysRevX.9.041001

Subject Areas: Atomic and Molecular Physics, Condensed Matter Physics

\section{INTRODUCTION}

Electronic transport in solids plays a fundamental role in our exploration of matter, and it constitutes the basis for innumerable device applications. In fact, the need for smaller and more efficient hardware has naturally led to the development of mesoscopic devices, where the quantum nature of the electron gas becomes relevant [1]. One of the most prominent examples of such quantum phenomena is the quantization of the electrical conductance in mesoscopic channels, which stems from the existence of discrete transverse modes [2-6]. In the 1980s, studies of the Hall conductance in two-dimensional electron gases subjected to high magnetic fields revealed the quantized Hall effect [7-9], which was later related to the existence of topological

\footnotetext{
*Corresponding author. grazia.salerno@ulb.ac.be

Corresponding author. ngoldman@ulb.ac.be

Published by the American Physical Society under the terms of the Creative Commons Attribution 4.0 International license. Further distribution of this work must maintain attribution to the author(s) and the published article's title, journal citation, and DOI.
}

invariants in the band structure [10,11] and chiral edge modes [12-15]. Moreover, such Hall measurements subsequently revealed the fractional quantum Hall effect [16], a first instance of a strongly correlated topological phase $[17,18]$. More recent transport experiments revealed the existence of topological insulators, such as those realizing the quantum spin Hall effect [19-22] as well as Dirac and Weyl semimetals $[23,24]$. In this regard, transport measurements are an important and well-established method for probing and studying the properties of quantum matter [25-27].

In parallel to the exploration of new materials and devices, quantum-engineered systems have been developed in ultracold-atom laboratories in view of offering a novel perspective on transport in quantum matter [28]. In these settings, nonequilibrium dynamics can be probed through different protocols [29], for instance, by suddenly releasing the atomic cloud in an optical lattice and imaging its expansion [30-32] or by driving the cloud with an external (optical or magnetic) force [33-38]. A third approach consists of engineering a two-terminal geometry, i.e., a mesoscopic channel for atoms connected to two reservoirs, using a constriction optical potential [39]: This scheme, which reproduces the two-terminal configuration used in 
electronic transport experiments, allows for a direct evaluation of a neutral gas' conductance. Importantly, such a setting has demonstrated the quantized conductance of a one-dimensional atomic channel [40], which constitutes a good starting point to study the transport of strongly correlated matter thanks to the ability to tune the interaction strength [41]. A particularly exciting perspective concerns the observation and characterization of fractional quantum Hall states in ultracold atomic gases [42-44].

Measuring the quantized Hall conductance of a twodimensional (2D) ultracold Fermi gas using the engineeredreservoir scheme of Refs. [39,40] is definitely appealing. However, this challenging goal would a priori require the combination of two main ingredients: (1) the realization of a synthetic gauge field $[42,45,46]$ to create a nontrivial topological band structure and reach the quantum Hall regime $[43,44]$, and (b) in direct analogy with the multiterminal devices (Hall-bar geometries) used in solid-state experiments [7-9], one would need to connect the 2D Fermi gas to several reservoirs. In this work, we propose that such an apparently complicated setting could in fact be readily designed starting from a single atomic channel [40] by exploiting the concept of synthetic dimensions [47-57]. As we show, the use of a synthetic dimension does not only simplify the implementation of the "atomic Hall bar," but it also allows for a direct readout of the quantized Hall conductance associated with chiral edge modes using a simple two-reservoir geometry. This scheme is universal in the sense that it could be applied to study the Hall conductance of a wide variety of atomic states (with potential applications to strongly correlated states). In addition, we note that the main concepts developed in this proposal could also be used in other physical platforms; see, for instance, the recent proposal in Ref. [58] to braid Majorana fermions in a single superconducting wire extended by a synthetic dimension; see also Refs. [59-62] for synthetic dimensions in photonics.

\section{A. The main approach and central results}

The aim of this work is to lay out a scheme by which a single atomic wire connected to two engineered reservoirs [Fig. 1(a)] can be turned into an atomic Hall bar: a 2D atomic system exhibiting the quantum Hall effect and designed so as to extract its (quantized) Hall conductance through transport.

Our approach is based on the observation that the constriction potential used to generate the atomic channel [39] defines a natural synthetic dimension. As illustrated in Fig. 1(b), the harmonic oscillator levels associated with the tight confinement form a large set of discrete states indexed by $\lambda \in \mathbb{N}$, which can be interpreted as fictitious "lattice sites" along a synthetic dimension. As shown in Ref. [56], motion can be induced along the $\lambda$ direction by shaking the channel in a time-periodic manner. Hence, within the central region of the constriction potential, atoms are
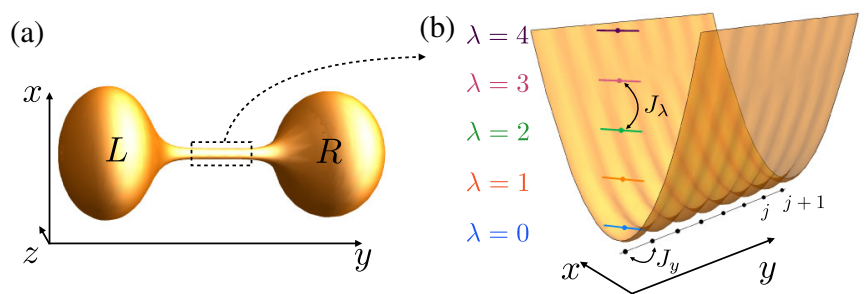

(c)

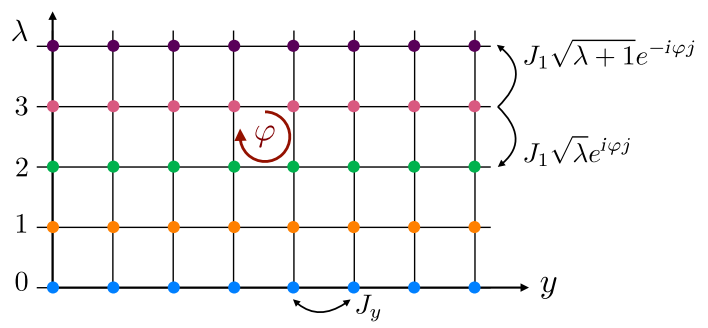

FIG. 1. (a) A sketch of the two-reservoir geometry [29], which is used as a basis for our shaken-channel model. A constriction potential is imprinted on a 3D Fermi gas so as to create a quantum wire, on top of which, a one-dimensional optical lattice is produced. The nonconstricted parts of the system form the two atomic reservoirs at the left $(L)$ and right $(R)$ sides of the channel. (b) A close-up view of the channel region shows the harmonic confinement associated with the constriction potential, as well as the optical lattice aligned along the channel; the corresponding sites are indexed by $j$ and the hopping amplitude denoted $J_{y}$. The harmonic oscillator levels $\lambda$ are reinterpreted as lattice sites along a synthetic dimension. The potential is then periodically shaken, as proposed in Ref. [56], to generate hopping processes along the synthetic dimension $\lambda$; the corresponding hopping amplitude is denoted $J_{\lambda}=J_{1} \sqrt{\lambda}$. (c) For a suitable shaking protocol, the system is described by a $2 \mathrm{D}$ tight-binding model in the $y-\lambda$ plane, which includes the effects of a tunable artificial magnetic field; the corresponding flux per plaquette is denoted by $\varphi$.

allowed to move along the real direction defined by the channel (denoted " $y$ direction" in Fig. 1 and hereafter), as well as along the synthetic dimension $\lambda$. We note that this construction essentially replaces the continuous transverse direction $x$ [Fig. 1(a)] by a discrete synthetic dimension $\lambda$ [Fig. 1(b)] and that the motion is inhibited along the third spatial direction $(z)$. In addition to this, we assume that the channel direction $(y)$ can also be discretized upon adding an optical lattice, as recently implemented in Ref. [63]. As a final ingredient, we assume that the phase of the modulation that generates motion along $\lambda$ can be made $y$ dependent, $\phi(y)$ : As previously shown in Ref. [56], this modification of the phase can generate a uniform magnetic flux in the 2D lattice defined in the fictitious $\lambda-y$ plane [Fig. 1(c)]; see also Refs. [64-68]. In the following, we consider that the reservoirs are not subjected to the modulation, and hence, that the corresponding regions do not include a synthetic magnetic field.

This work analyzes the conductance of this hybrid 2D atomic system, as probed by the inherent two-reservoir geometry (Fig. 1). At this stage, let us highlight a couple of 
peculiarities introduced by the synthetic $(\lambda)$ dimension. First, we emphasize that this synthetic dimension is intimately related to the energy of the system (each "site" along $\lambda$ is associated with a harmonic oscillator level), and hence, it cannot be simply treated as a genuine spatial direction. In particular, there is a built-in chemical-potential bias along the $\lambda$ direction, in the sense that particles privilege the occupation of low- $\lambda$ (i.e., low-energy) states; this natural bias leads to a subtle interplay with the overall chemical-potential imbalance that is imposed by the two reservoirs to drive current across the channel [Fig. 1(a)]. Second, since the system is periodically driven (and thus belongs to the class of Floquet-engineered systems [69-72]), transport properties rely on the underlying quasienergy spectrum [73-75]. Altogether, the population of the Floquet eigenstates associated with the inner-driven system is nonthermal, but it reflects the thermal population in the undriven reservoirs.

As we explain in more detail in the following sections, these unusual features lead to an effective (fictitious) multiterminal geometry, which allows us to substantially improve the conductance measurement stemming from the (real) two-reservoir geometry [Fig. 1(a)]. As a central result of our work, we demonstrate that a proper state preparation and reservoir configuration can allow for a clear separation of the bulk and edge contributions to the conductance. In particular, our unusual single-channel setup can be designed so as to fully resolve the quantized Hall conductance associated with chiral edge modes (we recall that this measurement requires at least four terminals in conventional static systems [8]). Our work opens new avenues towards the exploration of topological transport in ultracold-atom experiments, through the development of new probing schemes based on synthetic dimensions.

\section{B. Outline}

The paper is organized as follows: Section II reviews the notions that play an important role in the main part of our study. We discuss the transport properties of a simple 2D quantum Hall system, the Harper-Hofstadter model [76], with particular attention given to the main differences between the transport measurements that are performed using two-terminal and four-terminal geometries [8]. Section III A introduces the shaken-channel scheme; we discuss the emergence of a synthetic dimension [56], derive an effective time-independent model, and propose a possible implementation using the constriction potential of Fig. 1. The transport properties of the effective timeindependent model are studied in two different regimes: In Sec. III B, we make an approximation and map the model to the standard Harper-Hofstadter Hamiltonian; in Sec. III C, we relax this approximation and we show that the signatures of quantized transport survive. We also discuss how the energetic nature of the synthetic dimension naturally leads to an effective multiterminal geometry, which greatly enriches the measurement based on the (real) two-reservoir geometry. In Sec. IV, we consider the fulltime-dependent problem and apply a transport formalism that accurately takes the periodically driven nature of the system into account [73-75,77]. We study the two aforementioned regimes in Secs. IVA and IV B, respectively. Experimental considerations are briefly discussed in Sec. V, and conclusions are drawn in Sec. VI.

\section{REVIEW OF QUANTUM HALL TRANSPORT: APPLICATION TO THE HARPER-HOFSTADTER MODEL}

Our method builds on the equivalence between energyresolved two-terminal measurements on a driven onedimensional system and multiterminal measurements on a fictitious two-dimensional setup. This section presents the target model, namely, the Harper-Hofstadter model and its topological conductance properties, which serve as a benchmark for our protocol, as we detail in later sections.

We review the peculiar transport properties associated with the quantum Hall effect by applying the theoretical framework offered by the Landauer-Büttiker formalism, which was originally developed for calculating the conductance in solid-state systems $[5,6,25,26]$, to the Harper-Hofstadter model [76]. For a review of the Landauer-Büttiker formalism and the recursive Green's function (RGF) method that we use to calculate the conductance shown in our paper, see the Appendix A. A generalization of the RGF method [73-75,77], which is specifically tailored to treat timeperiodic systems, is presented in Appendix B.

The main goal of this section is to distinguish between the transport measurements that result from two-terminal and four-terminal geometries; we assume zero temperature throughout. We also discuss how the results of the RGF method can be interpreted in terms of (topological) edgestate transport based on the Landauer-Büttiker formalism $[78,79]$. The results of this section constitute a good basis for understanding the transport properties of the timeindependent shaken-channel model (Fig. 1), which approximately maps onto the Harper-Hofstadter model (see Sec. III B).

The Harper-Hofstadter Hamiltonian describes a particle moving in a two-dimensional lattice in the presence of a perpendicular magnetic field [76]

$\hat{H}=-J \sum_{i, j}\left(e^{i \varphi j}|i, j\rangle\langle i+1, j|+| i, j\rangle\langle i, j+1|+\right.$ H.c. $)$.

This Hamiltonian describes hopping processes taking place between nearest-neighboring sites $(i, j)$ of the lattice, where the indices $i$ and $j$ refer to the two directions ( $x$ and $y$ ), respectively. For fractional values of the flux $\varphi=$ $2 \pi p / q$, with $q, p \in \mathbb{Z}$, the spectrum of the Hamiltonian in Eq. (1) depicts $q$ bulk bands, which are connected by 
topologically protected chiral edge states [15]. These chiral edge states are responsible for the quantized Hall conductance of the system whenever the Fermi energy lies in a spectral bulk gap $[7,10,12,15]$. Specifically, in this quantum Hall regime, the longitudinal conductance vanishes, while the transverse (Hall) conductance exhibits robust plateaus [7], whose values directly correspond to the number of current-carrying edge states $[14,15]$. This behavior can be understood as the bulk of the system being insulating, while chiral edge currents carry the Hall current. Importantly, the chirality (orientation) of these edge modes around the 2D sample determines the sign of the Hall conductance.

We now discuss how these considerations apply to the conductance signal that is extracted from transport measurements using two or four terminals.

\section{A. Two-terminal geometry}

We consider a square lattice with $N_{x}$ and $N_{y}$ sites along the $x$ and $y$ directions, respectively. This lattice constitutes the inner system to be probed. Then, each site at the left and right end of the inner system is coupled to a reservoir through a left and right terminal, respectively. An example of such a geometry is shown in Fig. 2, where the inner system is represented in black, while the reservoir sites and the terminal links connecting them to the inner system are indicated in red. For simplicity, we indicate only a few sites of the reservoirs, which are assumed to be very large and to have translational symmetry (as is schematically indicated by dashed lines). The two reservoirs are labeled with $L$ and $R$, respectively, for left and right. We assume that a small bias is applied to the left reservoir and that the conductance $G_{L, R}$ is measured through a current detected at the right terminal. The linear dc current at zero temperature is

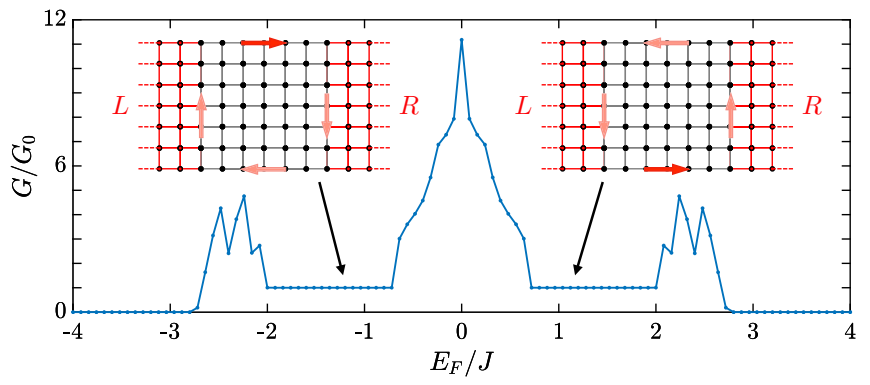

FIG. 2. (Insets) Example of a two-terminal setup with a square lattice geometry of $N_{x}=N_{y}=7$ sites along the $x$ and $y$ directions, respectively. The inner system is represented by the black dots, while the red dots identify the sites of the left $(L)$ and right $(R)$ reservoir. The arrows describe the motion of the chiral edge modes present in the model (clockwise at energies $E<0$ and counterclockwise at $E>0$ ). (Main) Conductance $G_{L, R}$ of the Harper-Hofstadter model with magnetic flux $\varphi=2 \pi / 3$, within the wideband approximation (see Appendix A), for an input bias applied to the left reservoir; the system size is $N_{x}=N_{y}=30$. The plateaus are associated with the edge modes illustrated in the insets.
$I_{L, R}=G_{L, R}\left(E_{F}\right)\left(\mu_{L}-\mu_{R}\right)$, where $G_{L, R}\left(E_{F}\right)$ is the conductance of the system at a given Fermi energy $E_{F}$, and $\mu_{L}$ $\left(\mu_{R}\right)$ is the chemical potentials of the $L(R)$ reservoir [25]. Following the Landauer formalism [5], the conductance is

$$
G_{L, R}\left(E_{F}\right) \equiv G_{0} \sum_{m} T_{L, R}^{(m)}\left(E_{F}\right),
$$

where the sum is over all possible transport channels and where we consider the case of spinless (single-component) fermions; here, $G_{0}=1 / h$ denotes the quantum of conductance, and we set $e=1$ to equally treat charged and neutral particles in this work. The transmission probability $T_{L, R}^{(m)}\left(E_{F}\right)$ is calculated from the scattering properties of the system following standard procedures, such as the RGF method [26,80-84]. More details are given in Appendix A.

The inner system is described by the Harper-Hofstadter model in Eq. (1), and we fix the flux per plaquette to the value $\varphi=2 \pi / 3$, for which the spectrum depicts three isolated bulk bands; in this setting, the two spectral gaps host a single chiral edge mode each [15]. The bulk gap at $E>0$ (resp. $E<0$ ) hosts an edge mode that propagates counterclockwise (resp. clockwise) around the 2D lattice. When the Fermi energy $E_{F}$ lies in the middle of a bulk band, one expects the observation of a metallic behavior: Bulk states provide a large set of nonperfectly transmitting channels, which results in a nonquantized conductance across the system. In contrast, when the Fermi energy is set within a band gap, the only channels that are available for transport are provided by the edge modes; in this regime, the conductance is quantized according to the number of edge modes present in the gap (i.e., one in the present model). Importantly, the chirality of the edge modes (and hence, the sign of the Hall conductance) cannot be identified in a two-terminal geometry [8]. Particles populating the "clockwise" (resp. "counterclockwise") chiral edge mode flow from the left to the right reservoir by following the top (resp. bottom) edge; see insets in Fig. 2. In both cases, this gives rise to a positive (quantized) conductance between the two reservoirs. In this sense, measuring a quantized conductance $G_{L, R}$ in this twoterminal geometry can reveal only the absolute value of the Hall conductance associated with the underlying 2D-lattice system [8]. We illustrate this phenomenon in Fig. 2, where we plot the conductance $G_{L, R}$ resulting from the RGF method as a function of the Fermi energy $E_{F}$ of the inner system (of size $N_{x}=N_{y}=30$ ). This plot clearly indicates that the conductance $G_{L, R}$ is quantized and positive whenever the Fermi energy falls within one of the two band gaps of the model (see the plateaus in Fig. 2), in agreement with the discussion above. Conversely, this same conductance is found to be not quantized whenever the Fermi energy hits one of the three bulk bands (see the irregular peaks in Fig. 2). Summarizing, while this twoterminal measurement cannot capture the chirality of the 
edge modes (and hence, the sign of the quantized Hall conductance), it does give a clear indication that the system displays perfectly transmitting channels (i.e., potential chiral edge modes) within well-defined energy ranges.

\section{B. Four-terminal geometry}

We now extend the two-terminal geometry by adding two more terminals at the top and at the bottom of the inner system; we label these $T$ and $B$, respectively. As a technical note, we impose that the corner sites of the inner system are coupled only to a single reservoir, which allows one to unambiguously define the terminal regions. An example of such a four-terminal geometry is shown in Fig. 3(a). As we further illustrate below, this four-terminal configuration allows for a clear and independent identification of the longitudinal and transverse (Hall) conductances of the system; we note that this configuration forms a minimal "Hallbar" setup, as routinely used in solid-state experiments.

Specifically, the longitudinal conductance $G_{\text {long }} \equiv G_{L, R}$ is obtained as in Sec. II A, namely, by applying a small bias to the left reservoir and measuring a current at the right terminal.

In order to extract the transverse (Hall) conductance $G_{\text {Hall }}$, one needs to analyze the transport that takes place between the top and the bottom reservoirs (while a bias is imposed at the left reservoir, as above). Applying the Landauer-Büttiker formalism to the four-terminal configuration $[85,86]$, one finds that the Hall conductance can be obtained as $G_{\text {Hall }} \equiv G_{L, T}-G_{L, B}$, which is indeed suitable in the present configuration where the bias is set in the left reservoir. We note that this expression for the Hall conductance is specifically chosen so as to probe the unidirectional transport of chiral edge states propagating around the 2D quantum Hall system [85].

In Fig. 3(b), we show the longitudinal conductance $G_{\text {long }}$ and the Hall conductance $G_{\text {Hall }} \equiv G_{L, T}-G_{L, B}$, as obtained
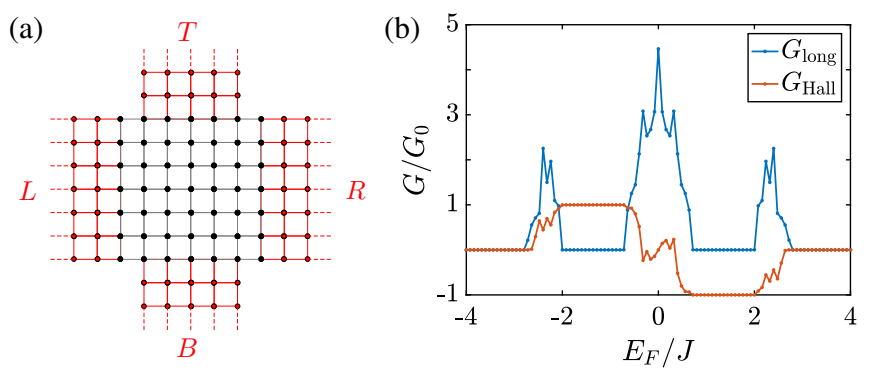

FIG. 3. (a) Example of a four-terminal setup with a square lattice geometry of $N_{x}=N_{y}=7$ sites along the $x$ and $y$ directions, respectively. The system is represented by the black dots, while the red dots identify the sites of the left $(L)$, right $(R)$, top $(T)$, and bottom $(B)$ reservoirs. (b) Longitudinal $\left(G_{\text {long }}=\right.$ $\left.G_{L, R}\right)$ and transverse Hall conductance $\left(G_{\text {Hall }}=G_{L, T}-G_{L, B}\right)$ of the Harper-Hofstadter model with magnetic flux $\varphi=2 \pi / 3$ for an input bias applied to the left reservoir; the system size is $N_{x}=N_{y}=30$. using the RGF method described in Appendix A. As before, the magnetic flux is $\varphi=2 \pi / 3$ in the inner system and the number of sites is $N_{x}=N_{y}=30$. In contrast with the result shown in Fig. 2, one now obtains a clear signature of the quantum Hall effect [7,8]: The longitudinal conductance vanishes, and the Hall conductance $G_{\text {Hall }}$ depicts clear plateaus whenever the Fermi energy $E_{F}$ falls within a band gap. In particular, the chirality of the propagating edge modes is now clearly identified through the sign of the (Hall) conductance.

Summarizing, a multiterminal setup (including at least four terminals) is required to unambiguously measure the quantized Hall conductance of a 2D system and to fully characterize the nature of the underlying chiral edge modes [8]. As we discuss below (Sec. III B), the energetic nature of the synthetic dimension that emerges from the shakenchannel system in Fig. 1 naturally leads to an effective multiterminal configuration, although the constriction potential creates only two atomic reservoirs: one on each side of the channel. This important observation is at the core of our present proposal.

\section{TRANSPORT IN A SHAKEN CHANNEL: THE EFFECTIVE-HAMILTONIAN APPROACH}

In this section, we introduce the mapping between the two-terminal conductance measurements on a driven onedimensional channel and the more conventional quantum Hall measurement as performed in two-dimensional systems. Specifically, our approach builds on (i) the mapping of a one-dimensional driven channel onto a fictitious twodimensional-lattice system via the concept of synthetic dimension [56]; (ii) the interpretation of the two nondriven reservoirs connected to the channel as many fictitious reservoirs connected along the synthetic dimension. From the latter interpretation, we find that there is a natural bias along the synthetic dimension, allowing for a direct measurement of a Hall-like response using a single channel.

\section{A. The model}

In this section, we define the shaken-channel model (Fig. 1) and describe its transport properties using an effectiveHamiltonian approach. We discuss how a natural synthetic dimension emerges in the problem and elaborate on how this feature affects the coupling to the reservoirs. In particular, this configuration leads to the notion of "effective multiterminal configurations," which allows for a clear detection of the quantum Hall effect in the shaken-channel model. The effective time-independent Hamiltonian approach is further validated in the full-time-dependent approach of Sec. IV.

\section{The shaken channel}

We consider a noninteracting gas of ultracold fermions (of mass $M$ ), which are restricted to move within a single channel aligned along the $y$ direction; we focus our 
attention on the channel and disregard the reservoirs for now. This system is described by a single-particle Hamiltonian of the form

$\hat{H}_{0}=\frac{\hat{p}_{x}^{2}}{2 M}+\frac{M \omega^{2}}{2} \hat{x}^{2}-J_{y} \sum_{j}[|x, j\rangle\langle x, j+1|+$ H.c. $]$.

The first two terms in Eq. (3) describe the motion along the harmonically confined transverse direction $(x)$, with trapping frequency $\omega$, while the last term describes motion along the channel. Here, we assume that a deep lattice potential is set along the channel and that a single-band tight-binding approximation can be made to capture the dynamics along this direction. Then, the hopping processes between neighboring orbitals $|x, j\rangle$ and $|x, j+1\rangle$ are fully characterized by the tunneling parameter $J_{y}$; here, $j=y / a$ refers to the site index along the channel direction, and we set the lattice spacing $a=1$ in the following.

Inspired by Ref. [56], we subject the tight harmonic confinement to a resonant time-periodic modulation, with frequency $\omega_{D} \approx \omega$,

$$
\hat{V}(t)=\kappa \hat{x} \cos \left(\omega_{D} t+\varphi j\right),
$$

which corresponds to shaking the channel along the transverse $(x)$ direction. Importantly, the modulation in Eq. (4) includes a phase $\theta(y)=\varphi j$, which explicitly depends on the channel direction $(y)$. In the following, we assume that such a modulation is active only on a (substantial) part of the channel, so that there are intermediate regions that adiabatically connect the nonshaken reservoirs to the shaken channel (i.e., the inner system).

Following Ref. [56], we write the total time-dependent Hamiltonian $\hat{H}(t)=\hat{H}_{0}+\hat{V}(t)$ in the basis formed by the harmonic oscillators states $|\lambda, j\rangle$, where $\lambda$ refers to the discrete harmonic levels associated with the transverse $(x)$ direction:

$$
\begin{aligned}
\hat{H}(t)= & \sum_{\lambda, j}\left[\omega \lambda|\lambda, j\rangle\langle\lambda, j|-\left(J_{y}|\lambda, j\rangle\langle\lambda, j+1|+\text { H.c. }\right)\right] \\
& +\sum_{\lambda, j} 2 \cos \left(\omega_{D} t+\varphi j\right) \\
& \times\left(J_{\lambda}|\lambda, j\rangle\left\langle\lambda+1, j\left|+J_{\lambda+1}\right| \lambda+1, j\right\rangle\langle\lambda, j|\right),
\end{aligned}
$$

where $J_{\lambda}=\kappa \sqrt{\lambda / 8 M \omega}$. In the high-frequency regime $\left(\omega_{D} \approx \omega \rightarrow \infty\right)$, an effective model can be obtained in the frame rotating at the shaking frequency by invoking the rotating-wave approximation $[56,70,72]$ :

$$
\begin{aligned}
\hat{H}_{\mathrm{eff}}= & \sum_{\lambda, j}\left[\Delta \lambda|\lambda, j\rangle\langle\lambda, j|-\left(J_{y}|\lambda, j\rangle\langle\lambda, j+1|+\text { H.c. }\right)\right] \\
& -\sum_{\lambda, j}\left(J_{\lambda} e^{i \varphi j}|\lambda, j\rangle\langle\lambda+1, j|+\text { H.c. }\right),
\end{aligned}
$$

where $\Delta=\omega_{D}-\omega$ is the detuning between the trap and drive frequencies; in the following, we consider a resonant drive with $\Delta=0$. As previously discussed in Ref. [56], the rotating-wave approximation and, hence, the description based on the effective Hamiltonian in Eq. (6), breaks down whenever $\left|J_{\lambda}\right| \gtrsim \omega / 4$. As a consequence, the number of states $\lambda$ that are available along the synthetic dimension for a given ratio $\kappa l_{H} / \omega$ is limited, where $l_{H}=1 / \sqrt{M \omega}$ is the harmonic length (which sets a natural length scale in the problem). Under these approximations, the effective timeindependent Hamiltonian in Eq. (6) corresponds to a 2D tight-binding model defined on a square lattice in the $\lambda-y$ plane, with inhomogeneous and anisotropic hopping strengths $\left(J_{\lambda}, J_{y}\right)$. Furthermore, this 2D-lattice model includes a uniform artificial magnetic field, which corresponds to having $\varphi$ quanta of flux per plaquette. Summarizing, the model realizes the Harper-Hofstadter model in Eq. (1), with inhomogeneous and anisotropic hopping parameters. We note that the optical lattice set along the channel direction is not a crucial ingredient, as removing it would result in a model of "quantum Hall wires" with similar properties $[87,88]$.

\section{Connecting the shaken channel to reservoirs}

Our proposal builds on the observation that the shaken channel described above is naturally connected to two reservoirs, as illustrated in Fig. 1. Specifically, the total system is constituted of an inner 2D system realizing the anisotropic Harper-Hofstadter model (defined in the fictitious $\lambda-y$ plane), which is connected to two nonshaken reservoirs. Note that these two reservoirs are attached at both ends of the channel; namely, they connect to the 2D inner system at $y=0$ and $y=L_{y}$.

In the following, we consider that a chemical-potential imbalance can be applied between the two reservoirs, so as to force the atoms to move from the left side of the channel to the right side (Sec. II); the relative particle difference then provides a measurement of the system's conductance [29]. Since the effective model describing the 2D inner system corresponds to the Harper-Hofstadter-like model in Eq. (6), one expects that the resulting transport properties should be reminiscent of those presented in Sec. II A (which discusses the conductance of the Harper-Hofstadter model as probed by a two-terminal geometry).

This naive prediction is based on the assumption that the synthetic $(\lambda)$ direction can be treated as a genuine spatial direction: Specifically, it assumes that the reservoirs inject particles in the inner system at $y=0$ in a $\lambda$-independent manner. However, the time-modulated (shaken) channel maps onto the model in Eq. (6) in a frame rotating at the shaking frequency, while the reservoirs have a thermal distribution in the laboratory frame. The transport measurement therefore needs to account for the fact that, in the laboratory frame, the $\lambda$ direction is the energy axis and the channel is explicitly time dependent. As we show below, 
the energetic nature of the synthetic dimension actually provides a novel route for the investigation of the topological features of the Harper-Hofstadter $(\mathrm{HH})$ model.

\section{B. Simple effective model: Homogeneous hopping along the synthetic dimension}

For the sake of clarity, let us first simplify the analysis of the time-independent effective model in Eq. (6) by neglecting the inhomogeneity in the hopping along the synthetic dimension $\lambda$; specifically, we substitute $J_{\lambda} \rightarrow J_{1} \equiv$ $\kappa \sqrt{1 / 8 M \omega}$. The corresponding effective model that describes the inner system then reads

$$
\begin{aligned}
\hat{H}_{\mathrm{eff}}^{\mathrm{HH}}= & -J_{y} \sum_{\lambda, j}(|\lambda, j\rangle\langle\lambda, j+1|+\text { H.c. }) \\
& -J_{1} \sum_{\lambda, j}\left(e^{i \varphi j}|\lambda, j\rangle\langle\lambda+1, j|+\text { H.c. }\right),
\end{aligned}
$$

and it exactly maps onto the Harper-Hofstadter model when $J_{y}=J_{1}$. We note that the approximation of constant $J_{\lambda}$ is valid only in the limit of large $\lambda \gg 1$, which is a priori problematic since we anticipate that low- $\lambda$ states should substantially contribute to transport (we remind the reader that transport is dominated by chiral edge modes in the quantum Hall regime and that $\lambda=0$ defines a clear edge along the synthetic dimension). In this sense, the results presented in this section aim to provide only a general intuition on the transport that takes place in this synthetic 2D system. The analysis of the full (inhomogeneous) effective Hamiltonian is postponed to Sec. III C, while the properties of the full-time-dependent model (including the application of the Floquet-Landauer approach to transport) are presented in Sec. IV.

Although the synthetic direction $\lambda$ introduced above is semi-infinite, we note that, in practice, the anharmonicity of the confinement realized in ultracold-atom experiments can produce a natural (soft) boundary. In addition, a box-type potential could be applied on top of the harmonic trap to further confine the particles along the transverse direction, which would result in a sharp edge in the $\lambda$ direction (at a desired $\lambda_{\text {cut }}$ ). In the following, we introduce a cutoff along the synthetic direction $\lambda_{\text {cut }} \gg 1$, which is convenient for performing numerical simulations on a finite system.

\section{Two-terminal geometry: Adding details to the reservoirs}

As a first step, we treat the synthetic dimension as a genuine spatial dimension. Under this assumption, the conductance of the model in Eq. (7) can be calculated as in Sec. II A, namely, by treating the full system (inner part and reservoirs) as a standard two-terminal problem. A sketch of this simple configuration is shown in the inset of Fig. 4(a).
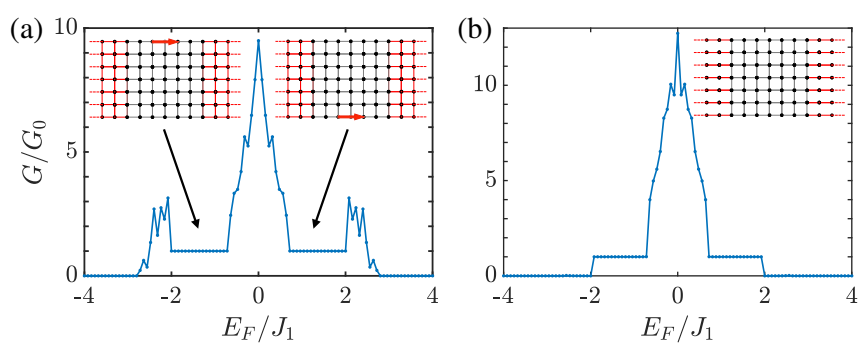

FIG. 4. Two-terminal conductance of the isotropic effective model in Eq. (7). Parameters are $\kappa=0.01 \omega / l_{H}, J_{y}=J_{1}$, $\varphi=2 \pi / 3, \lambda_{\text {cut }}=29, N_{y}=30$. The sites in the reservoirs are always horizontally coupled $J_{y}^{r}=J_{y}$, while vertical couplings in the reservoirs are (a) $J_{1}^{r}=J_{1}$, such that the wideband approximation is valid, or (b) $J_{1}^{r}=0$, for which the wideband approximation breaks down.

We now check whether the details of the reservoirs influence the calculation of the conductance. This is different from applying the wideband approximation described in Appendix A, as is considered for the calculation shown in Fig. 2. As a first assumption, we take the Hamiltonian describing the reservoir as in Eq. (7) but with $\varphi=0$; i.e., the effective magnetic field is assumed to be absent in the reservoirs. Furthermore, we first (naively) assume that the hopping amplitudes are uniform and isotropic throughout the entire system: $J_{y}^{r}=J_{y}$ and $J_{1}^{r}=J_{1}$, where the superscript $r$ refers to the reservoirs. The resulting conductance plot is shown in Fig. 4(a), which naturally shares the same features as those previously displayed in Fig. 2 (Sec. II A). In particular, the plateau at $E_{F}>0$ in Fig. 4(a) is attributed to the counterclockwise propagating edge mode, which is associated with the spectrum of the Harper-Hofstadter Hamiltonian in Eq. (7), and which is localized at $\lambda=0$. Conversely, the plateau at $E_{F}<0$ is associated with the clockwise propagating edge mode localized at $\lambda=\lambda_{\text {cut }}$; see inset of Fig. 4(a).

We stress that the description used above for the reservoirs $\left(J_{y}^{r}=J_{y}\right.$ and $\left.J_{1}^{r}=J_{1}\right)$ is not compatible with the actual scheme described in Sec. III A. Indeed, since the temporal modulation acts only on the inner part of the system (or more precisely, on a substantial part of the transport channel), a more accurate description consists of setting $J_{1}^{r}=0$ in the junction regions connecting the inner system to the reservoirs (noting that the harmonic oscillator states $\lambda$ are indeed decoupled in the absence of the time modulation); see the sketch in the inset of Fig. 4(b). As we show in the conductance plot of Fig. 4(b), the quantized plateaus remain unaffected by this modification of the reservoirs' properties [compare Figs. 4(a) and 4(b)].

As a technical remark, we note that the bulk-band response at $\left|E_{F} / J_{1}\right|>2$ is dramatically suppressed in Fig. 4(b), which is due to the breakdown of the wideband approximation: When setting $J_{1}^{r}=0$, the bandwidth associated with the reservoirs is of the order of $W^{r} \approx 2 J_{y}^{r}$, which is smaller than the bandwidth of the inner system, 
$W=4 J_{1}=4 J_{y}^{r}$, in the situation considered in Fig. 4(b). We check that the bulk response of Fig. 2 is indeed recovered when setting $J_{y}^{r} \gtrsim J_{y}=J_{1}$, i.e., when reaching a regime where the wideband approximation is again satisfied. Importantly, we verify that the details of the reservoirs do not break the robustness of the quantized plateaus, as soon as the wideband approximation is fulfilled. In the remainder of the paper, we always treat the reservoirs in the wideband approximation.

\section{Effective multiterminal geometry}

As a crucial step in the description and understanding of our scheme, we now take the energetic nature of the synthetic dimension into account. To do so, we analyze how particles are injected from the left reservoir into the inner shaken channel by partitioning the whole system into three connected parts: (a) the inner system (2D lattice in the fictitious $\lambda-y$ plane), (b) the two reservoirs, (c) the two junction regions that connect the inner system to the reservoirs; see Fig. 5. It is reasonable to assume that the junction regions can be treated as discrete harmonic levels of energy $\hbar \omega$, which are populated following a thermal (Fermi-Dirac) distribution set by the reservoirs: Particles in a given $\lambda$ state are injected if $\lambda \hbar \omega<E_{F}$, and holes are injected for $\lambda \hbar \omega>E_{F}$. In this sense, the reservoir injects more particles in the low- $\lambda$ states (bottom of the hybrid 2D inner system) than in the high- $\lambda$ states: The reservoir is not "connected" uniformly along the synthetic dimension, and there is an effective chemical-potential bias along the

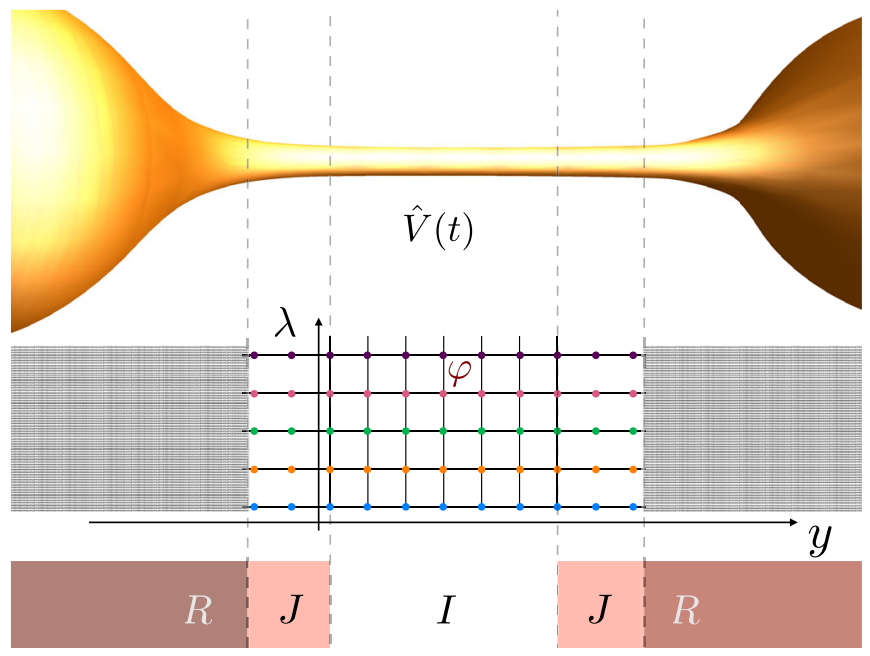

FIG. 5. Connecting the fictitious 2D system to two reservoirs: (I) the inner system where the time modulation $\hat{V}(t)$ is applied (resulting in a fictitious 2D lattice defined in the $\lambda$-y plane), $(R)$ the two reservoirs, and $(J)$ the two junction regions (where coupling between the $\lambda$ states is absent). Particles leave a reservoir to enter a nonshaken junction region (where the populations of the $\lambda$ states rely only on the Fermi-Dirac distribution associated with the reservoir); particles then leave the junction to enter the inner system in an adiabatic manner.

$\lambda$ direction. A simple way to include this unusual feature in our effective-Hamiltonian description consists of splitting up the left and right reservoirs into many (fictitious) reservoirs, all aligned along the synthetic $\lambda$ direction with varying chemical potentials (depending on their location along the synthetic dimension); it is the aim of the two following paragraphs to study the resulting transport properties. We point out that this effective bias along the $\lambda$ direction can be naturally controlled experimentally via the overall chemical potential and temperature of the reservoirs.

As before, we still assume that the chemical potentials are set such that transport is driven from the left part to the right part of the system. In addition, in the following paragraphs, we assume that hopping is allowed in the reservoirs along the synthetic dimension $\left(J_{1}^{r}=J_{1}\right)$; this choice can be modified in order to reach an even finer description (i.e., by setting $J_{1}^{r}=0$; see discussion of Sec. III B 1). Other transport configurations are also briefly discussed below.

Effective four-terminal geometry.-As a first approximation, we consider that the initial two-reservoir configuration can be split into an effective four-terminal geometry: The inner system is coupled to two effective reservoirs on the left (labeled by $\left.L_{1,2}\right)$ and by two effective reservoirs $\left(R_{1,2}\right)$ on the right; see the sketch in Fig. 6(a). We point out that this setting corresponds to a rearrangement of the more standard four-terminal geometry previously discussed in Sec. II B [Fig. 3(a)]. Importantly, it turns out that this unusual (effective) four-terminal geometry allows for a clear measure of the Hall and longitudinal conductances, as we now explain.

(a)

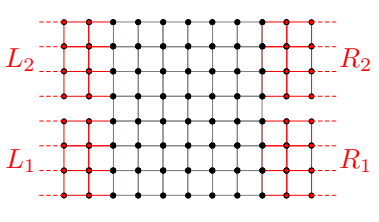

(c)
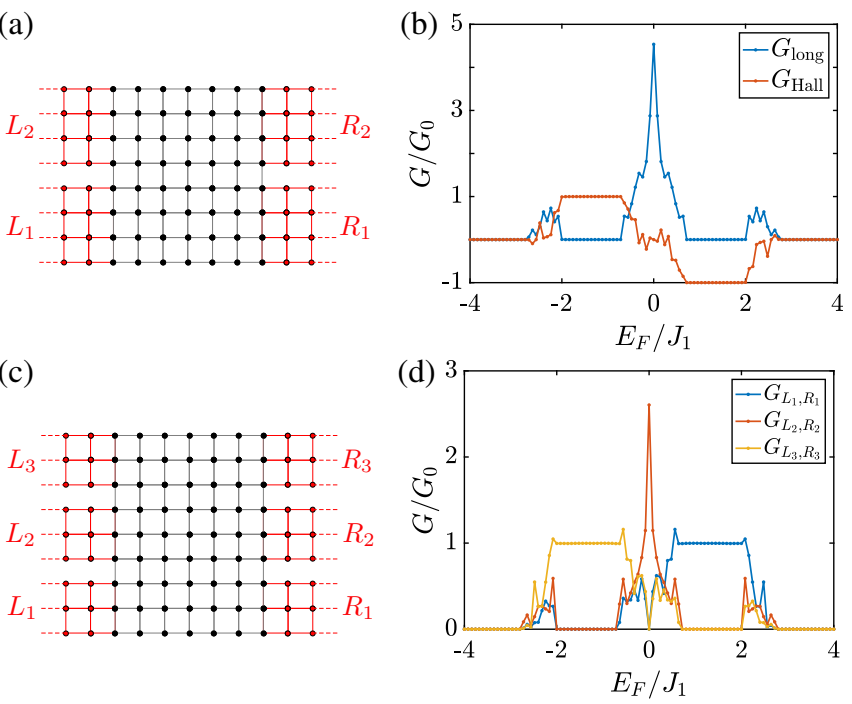

FIG. 6. (a) Effective four-terminal geometry and (b) the resulting longitudinal and Hall conductances. (c) Effective six-terminal geometry and (d) the resulting conductances $G_{L_{i}, R_{i}}$ for $i=1,2,3$. Parameters are $\kappa=0.01 \omega / l_{H}, J_{y}=J_{1}, \varphi=2 \pi / 3, \lambda_{\text {cut }}=29$, $N_{y}=30$. 
Figure 6(b) shows the conductances as obtained from the RGF method for two different configurations: The longitudinal conductance is calculated as $G_{\text {long }} \equiv G_{L_{1}, R_{2}}$, while the transverse (Hall) conductance is evaluated as $G_{\text {Hall }} \equiv G_{L_{2}, R_{2}}-G_{L_{1}, R_{1}}$. These choices can be explained based on simple arguments. First, as previously discussed in Sec. II B, the longitudinal conductance results from the contribution of the many extended bulk states, and since the transport taking place between the terminals $L_{1}$ and $R_{2}$ in Fig. 6(a) necessarily involves bulk states, it is thus legitimate to define the longitudinal conductance as $G_{L_{1}, R_{2}}$ in this context. Second, in quantum Hall systems, the Hall conductance can be attributed to the contribution of the edge modes. We note that the transport between the terminals $L_{1}$ and $R_{1}$ in Fig. 6(a) can be attributed to the propagation of a counterclockwise edge mode along the bottom edge as well as to bulk states and similarly that the transport between the terminals $L_{2}$ and $R_{2}$ involves the bulk and a clockwise edge state following the top edge. Consequently, the difference $G_{L_{2}, R_{2}}-G_{L_{1}, R_{1}}$ allows one to reveal the edge-current contribution, and thus, the Hall conductance; a more rigorous derivation can be obtained based on the Landauer-Büttiker formalism $[85,86]$.

Effective six-terminal geometry.-One can further refine the model by considering an effective six-terminal configuration, where the main (physical) reservoirs are now split into three effective reservoirs each; we denote these $L_{1,2,3}$ on the left and $R_{1,2,3}$ on the right, respectively; see the sketch in Fig. 6(c). As compared to the four-terminal configuration [Fig. 6(a)], the six-terminal geometry allows for an even more direct detection of the bulk and edge contributions to transport. Indeed, $G_{L_{2}, R_{2}}$ reflects the transport taking place in the bulk and hence provides an accurate probe of the longitudinal conductance, whereas $G_{L_{1}, R_{1}}$ (resp. $G_{L_{3}, R_{3}}$ ) reflects the transport associated with the counterclockwise (resp. clockwise) edge mode propagating along the bottom (resp. top) edge. These contributions are demonstrated in Fig. 6(d), which shows the corresponding conductances and which indeed reproduce the expected features of the Hall and longitudinal conductances. As a technical remark, we note that $G_{L_{1}, R_{1}}$ and $G_{L_{3}, R_{3}}$ also show a weak contribution of the bulk states in the vicinity of the band edges.

This construction of an effective multiterminal geometry can be straightforwardly extended to the limit where each row of sites at a given $\lambda$ is connected to a terminal $L_{\lambda}$ (resp. $R_{\lambda}$ ) on the left (resp. right); see Fig. 7(c). In this extreme case, the conductances $G_{L_{\lambda=0}, R_{\lambda=0}}$ and $G_{L_{\lambda=\lambda_{\text {cut }}}, R_{\lambda=\text { cut }}}$ would isolate the chiral-edge-mode contributions, while the others $G_{L_{\lambda}, R_{\lambda}}$ would capture the contributions from the bulk states only.

Other configurations.-We point out that other transport configurations can be envisaged. For instance, the motion
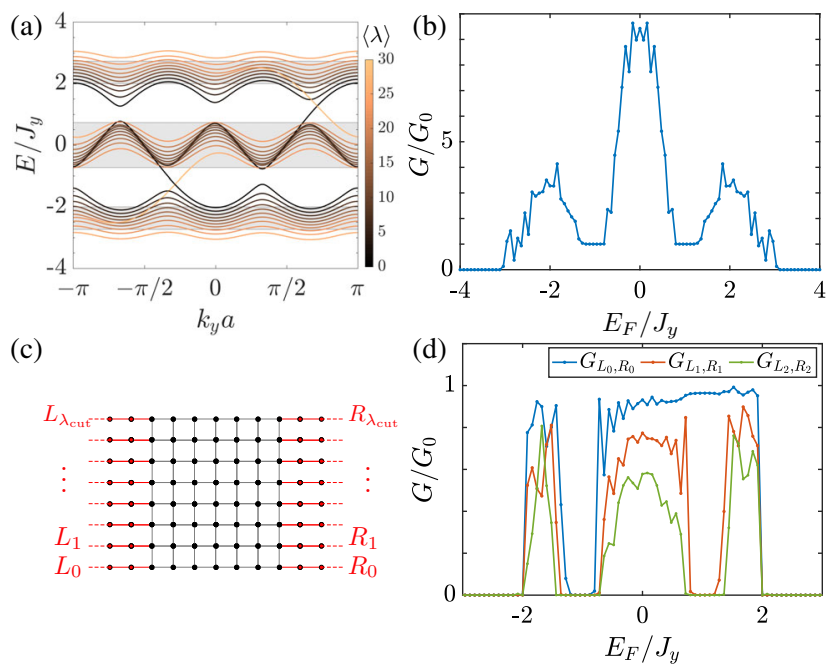

FIG. 7. (a) Energy dispersion of the complete effective model with $\lambda$-dependent hopping [Eq. (6)] for periodic boundary conditions along $y$ and $\lambda_{\text {cut }}=30$. The color scale indicating the mean position $\langle\lambda\rangle$ of the eigenstates along the synthetic dimension is chosen so as to highlight low- $\lambda$ states (darker colors). The shaded regions correspond to the bulk bands of the homogeneous and isotropic model of Sec. III B. (b) Two-terminal conductance of the complete effective model in Eq. (6). (c) Sketch of the effective multiterminal geometry with $N=0, \ldots, \lambda_{\text {cut }}$, and (d) the resulting conductances $G_{L_{\lambda}, R_{\lambda}}$ for $\lambda=0,1,2$. Parameters are $\kappa=0.01 \omega / l_{H}, J_{y}=4 J_{1}, \varphi=2 \pi / 3, \lambda_{\text {cut }}=30$.

taking place along the synthetic dimension could be probed by analyzing the conductance associated with two fictitious terminals located on a given side of the channel (e.g., $G_{L_{\lambda}, L_{\lambda^{\prime}}}$ or $G_{R_{\lambda}, R_{\lambda^{\prime}}}$ ). Such a motion along $\lambda$ would physically correspond to a heat transport [89] within a given (real) reservoir. In addition, the detuning $\Delta$ defined in Eq. (6) could be used to generate an artificial electric field aligned along the synthetic dimension, hence, offering an additional control parameter to the transport experiment.

\section{Complete effective model with $\lambda$-dependent hopping}

So far, we have described the shaken-channel model in terms of the simplified effective Hamiltonian in Eq. (7), namely, the Harper-Hofstadter model (Sec. II) with isotropic and homogeneous hopping $\left(J_{y}=J_{1}\right)$. This allows us to analyze how conductance measurements are modified as one changes the reservoirs' configuration, offering a first important step in our understanding of how transport takes place in the presence of a synthetic dimension (Sec. III B).

We now go beyond these studies and consider the complete effective model in Eq. (6) by taking the inhomogeneous hopping along the synthetic dimension $\left(J_{\lambda}\right)$ into account; as before, we take the resonant-driven limit and set $\Delta=0$.

As a first step, we calculate the energy spectrum for this effective model in view of identifying the energy ranges 
that correspond to the edge modes (and bulk gaps); these ranges will then correspond to the quantized plateaus depicted by the Hall conductance when plotted as a function of the Fermi energy. Following Ref. [56], we diagonalize the Hamiltonian in Eq. (6) in a gauge where translational symmetry is recovered along the $y$ direction. Applying periodic boundary conditions along the $y$ direction and setting $\lambda_{\text {cut }}=30$, one obtains the spectrum shown in Fig. 7(a); the color scale indicates the mean position $\langle\lambda\rangle$ of the eigenstates along the synthetic dimension. The main effect of the inhomogeneous and anisotropic hopping is to increase the bandwidth of the spectrum and to reduce the size of the bulk gap that hosts the topological edge modes. The bandwidth of the complete effective model can be compared with the one of the homogeneous and isotropic model indicated as gray regions in Fig. 7(a). For our choice of parameters $J_{y}=4 J_{1}$, these modifications are not too severe in the sense that chiral edge modes are still present in reasonably large bulk gaps (of order $J_{y}$ ); we also note that the group velocity (and chirality) of the edge modes is still preserved. For example, the edge mode located in the upper gap, and which propagates from left to right (positive group velocity along $y$ ), is localized at $\lambda=0$, while in the lower gap, this mode is localized at $\lambda=\lambda_{\text {cut }}$.

We now calculate the conductance of this effectiveHamiltonian system based on a simple two-terminal configuration, which allows for a direct comparison with the results previously presented in Fig. 4(a); hence, for clarity, we first neglect the energetic nature of the synthetic dimension in this part of the study. The results are presented in Fig. 7(b), which shows the conductance calculated using the RGF method. Importantly, the plateaus associated with the chiral edge modes (at $\lambda=0$ and $\lambda=\lambda_{\text {cut }}$ ) are still visible in this more realistic (anisotropic) model. We also notice that the size of the plateaus, which is indicative of the band gaps displayed in Fig. 7(a), is reduced compared to the isotropic case shown in Fig. 4(a).

Finally, one can include the energetic nature of the synthetic dimension into the description by following the effective-multiterminal construction of Sec. III B 2 shown in Fig. 7(c). The conductance $G_{L_{\lambda}, R_{\lambda}}$ for the lowest three terminals $\lambda=0,1,2$ is plotted in Fig. 7(d), which indicates that the edge modes of the realistic model can be unambiguously identified through the multiterminal geometry of Sec. III B 2.

\section{FULL-TIME-DEPENDENT PROBLEM: A FLOQUET-LANDAUER APPROACH TO TRANSPORT}

In the previous sections, we analyze the conductance of the shaken-channel model using an effective-Hamiltonian approach; there, traditional tools of quantum transport are directly applied to time-independent Hamiltonians, which allow us to explore the peculiarities introduced by the synthetic dimension. In particular, we discuss how the emergent notion of an effective multiterminal configuration allows for a clear detection of the transverse (Hall) and longitudinal conductances in a single atomic wire, hence, revealing the quantum Hall effect in a minimal cold-atom setting.

In this section, we now build on these results to analyze the full-time-dependent problem using the Floquet-Landauer approach to transport that we describe in Appendix B. The aim of this section is to fully validate the main result of this article, namely, that the quantized Hall conductance associated with chiral edge modes can be extracted from the shaken-channel model displayed in Fig. 1.

Consider the Schrödinger equation associated with the full time-dependent Hamiltonian in Eq. (5), which is expressed in the basis of the harmonic oscillator states:

$$
\begin{aligned}
i \partial_{t} \psi_{\lambda, j}(t)= & \sum_{\lambda, j}\left[\omega \lambda \psi_{\lambda, j}(t)-J_{y}\left(\psi_{\lambda, j+1}(t)+\psi_{\lambda, j-1}(t)\right)\right] \\
& +\sum_{\lambda, j} 2 J_{1} \cos (\omega t+\varphi j) \\
& \times\left(\sqrt{\lambda} \psi_{\lambda-1, j}(t)+\sqrt{\lambda+1} \psi_{\lambda+1, j}(t)\right),
\end{aligned}
$$

where $\psi_{\lambda, j}(t)$ is the wave function of a particle in the state $|\lambda, j\rangle$; as previously, we explicitly set the drive frequency on resonance $\omega_{D}=\omega$. The time-periodic system in Eq. (8) is associated with a quasienergy (Floquet) spectrum, which can be defined in a restricted range $\varepsilon \in[-\omega / 2, \omega / 2]$; this spectrum can also be represented in an extended-zone scheme $E=\varepsilon+n \omega$, where the integer $n$ refers to the repeated multiplicities. As discussed in Ref. [90], it is convenient to treat such periodically driven systems in an extended (Floquet) Hilbert space, which explicitly takes these multiplicities into account. We then expand the wave function into its Fourier components,

$$
\psi_{\lambda, j}(t)=\sum_{n=-n_{F}}^{n_{F}} \psi_{\lambda, j}^{(n)} e^{i n \omega t}
$$

where we truncate the series up to $2 n_{F}+1$ modes, and where $\psi_{\lambda, j}^{(n)}$ denotes the time-independent Fourier amplitudes; the number of modes $n_{F}$ can be chosen so as to reach convergence of the numerical observables. Substituting Eq. (9) into Eq. (8) and isolating the components proportional to $e^{i n \omega t}$, we obtain the Fourier component $\mathbf{H}_{n}$ of the Hamiltonian in Eq. (5) (see Appendix B):

$$
\begin{aligned}
& \omega(\lambda+n) \psi_{\lambda, j}^{(n)}-J_{y}\left(\psi_{\lambda, j+1}^{(n)}+\psi_{\lambda, j-1}^{(n)}\right) \\
& \quad+J_{1} e^{i \varphi j}\left(\sqrt{\lambda} \psi_{\lambda-1, j}^{(n-1)}+\sqrt{\lambda+1} \psi_{\lambda+1, j}^{(n-1)}\right) \\
& \quad+J_{1} e^{-i \varphi j}\left(\sqrt{\lambda} \psi_{\lambda-1, j}^{(n+1)}+\sqrt{\lambda+1} \psi_{\lambda+1, j}^{(n+1)}\right) \equiv \mathbf{H}_{n} \psi_{\lambda, j}^{(n)} .
\end{aligned}
$$


At this stage, the only approximation that is used concerns the truncation of the Fourier space into $2 n_{F}+1$ modes. In particular, no assumption is made on the frequency of the shaking, which implies that Eq. (10) is valid beyond the rotating-wave approximation (i.e., in the regimes of slow time modulation). However, in the following we set the system parameters within the range of validity of the rotating-wave approximation, which allows for a good comparison between the results obtained from the fulltime-dependent Hamiltonian in Eq. (10) and those stemming from the effective model defined in Sec. III A. Moreover, we point out that Eq. (10) explicitly involves the energy $\omega \lambda$, which indicates that the energetic nature of the synthetic dimension is implicitly present in the description.

We now apply the methods described in Appendix B to numerically compute the conductance of the system described by Eq. (10) by generalizing Eq. (2) for timeperiodic systems. Because of the driven nature of the system, it is useful to define a "Fermi quasienergy" $\varepsilon_{F} \in$ $[-\omega / 2, \omega / 2]$ such that $E_{F}=\varepsilon_{F}+n \omega$, where $E_{F}$ refers to the Fermi energy set by the unshaken reservoirs. As discussed in Refs. [75,91], the conductance of the effective model is then recovered by summing the transmissions $T_{\alpha, \beta}^{(m)}$ attributed to the different Floquet multiplicities

$$
T_{\alpha, \beta}^{(m)}\left(\varepsilon_{F}\right)=\sum_{n \in \mathbb{Z}} T_{\alpha, \beta}^{(m)}\left(\varepsilon_{F}+n \omega\right),
$$

which is then to be combined with Eq. (2). The Floquet sum rule in Eq. (11) allows one to recover the conductance of the effective (Floquet) time-independent model [75].

Specifically, the conductance is calculated by considering a two-terminal geometry, namely, by explicitly using the fact that the shaken channel is physically connected to two reservoirs (Fig. 1). As we state above, we stress that the energetic nature of the synthetic dimension is naturally included in the formalism through Eq. (10). The next paragraphs demonstrate how this full-time-dependent approach reproduces the features that were previously obtained based on the effective-Hamiltonian (multiterminal) approach described in Sec. III B 2.

\section{A. Time-dependent model with homogeneous hopping}

As a first step, we propose to analyze the transport properties of the full-time-dependent model by supposing that all the hopping processes in Eq. (10) are uniform and isotropic over the entire (fictitious) 2D lattice (see Sec. III B). In this way, one will be able to directly compare the results obtained from the Floquet-Landauer approach with those previously presented in Sec. III B 1 (where the effective-Hamiltonian approach is studied based on a simple two-terminal geometry); in particular, this first analysis will shed some light on the Floquet sum rule [Eq. (11)] in the context of a time-dependent twoterminal setup.
The results presented in this section are obtained using the Floquet RGF method described in Appendix B; in the present model, numerical convergence is reached for $n_{F}=\lambda_{\text {cut }}$, where $\lambda_{\text {cut }}$ denotes the cutoff along the synthetic dimension.

We illustrate the results in Fig. 8(a), which shows the conductance of the shaken-channel [Eq. (8)] with $N_{y}=12$ lattice sites along the real direction $y$, and we set $\lambda_{\text {cut }}=11$. Here, we apply the Floquet sum rule by including the contribution of all Fourier modes [Eq. (B8)], which, as discussed in Ref. [75], allows for an accurate evaluation of the conductance associated with the underlying effective (time-independent) system. In the present case, including all these contributions leads to the clear quantized plateaus in Fig. 8(a), in agreement with the effective-Hamiltonian result in Fig. 4.

As a technical remark, we note that the results are obtained within the validity range of the wideband approximation (see Appendix A). In the context of time-periodic systems, this approximation should be extended by assuming that the self-energy and the linewidth are energy independent for all the Floquet multiplicities that contribute to the sum rule in Eq. (11); as discussed in Ref. [75], this is required to accurately capture the conductance.

\section{Identification of chiral edge modes using the Floquet sum rule}

As we illustrate above, the Floquet sum rule [Eq. (11)] allows one to evaluate the conductance associated with the underlying effective model $[75,91]$. In order to test this result, we show in Fig. 8(b) the conductance as obtained by truncating the sum over the Fourier modes up to some critical mode $n_{\text {cut }}:$ By restricting this sum to $n=-n_{F}, \ldots, 5$, we find that the plateau at $\varepsilon_{F}<0$ is drastically reduced, while the quantized plateau at $\varepsilon_{F}>0$ survives the truncation [compare the blue curve in Fig. 8(b) with Fig. 8(a)]. In addition, we find that this behavior is reversed (i.e., only
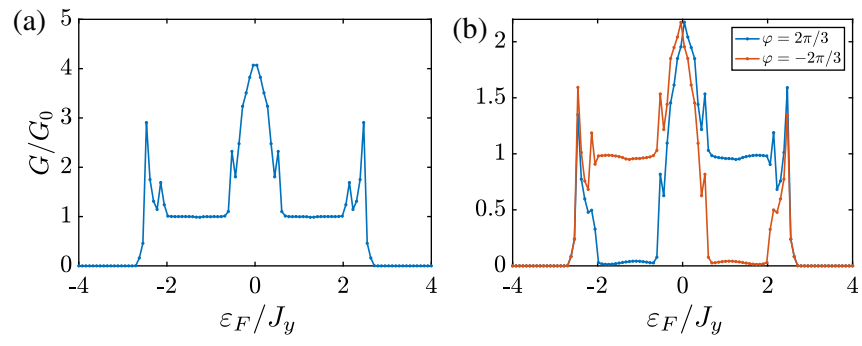

FIG. 8. Conductance of the shaken-channel model described by Eq. (8) when neglecting the anisotropy in the hopping, as obtained from the Floquet RGF method and Floquet sum rule in Eq. (11). Parameters are $\lambda_{\text {cut }}=11, N_{y}=12, \varphi=2 \pi / 3$, $J_{y}=J_{1}, \kappa=0.03 \omega / l_{H}$, and $n_{F}=11$. (a) The sum in Eq. (11) is taken over all the Floquet modes, from $n=-11$ up to $n=n_{F}=11$; (b) the sum is performed up to the $n=5$ component. 
the plateau at $\varepsilon_{F}<0$ survives) upon reversing the sign of the magnetic flux $(\varphi=-2 \pi / 3)$. This suggests an interesting interplay between the truncation of the Floquet sum rule, the energetic nature of the synthetic dimension, and the detection of edge modes, as we now explain.

From our analysis of the effective Hamiltonian, we know that the quantized plateau at $\varepsilon_{F}>0$ (resp. $\varepsilon_{F}<0$ ) is due to the edge mode localized at $\lambda=0$ (resp. $\lambda=\lambda_{\text {cut }}$ ). When the Floquet sum rule is truncated up to some mode $n_{\text {cut }} \ll \lambda_{\text {cut }}$, the total transmission $\sum_{n<n_{\text {cut }}} T\left(\varepsilon_{F}+n \omega\right)$ no longer captures the contribution of the edge mode localized at $\lambda=\lambda_{\text {cut }}$; this explains the absence of the expected plateau at $\varepsilon_{F}<0$ in Fig. 8(b) (blue curve). In contrast, the quantized plateau at $\varepsilon_{F}>0$ is still present, since the total transmission still captures the contribution of the edge mode localized at $\lambda=0$. This observation is further validated by reversing the magnetic flux $(\varphi=-2 \pi / 3)$ : In this case, the propagating edge mode at $\lambda=0$ corresponds to the lower gap $\varepsilon<0$, and hence, it is the plateau at $\varepsilon_{F}>0$ that disappears [red curve in Fig. 8(b)].

This apparent relation between the Fourier modes $(n)$ entering the Floquet sum rule and the site index $\lambda$ associated with the synthetic dimension naturally stems from the resonant nature of the time modulation, which is at the heart of the present proposal. Indeed, the harmonic oscillator levels (i.e., the sites along the synthetic dimension $\lambda$ ) are equispaced according to the energy separation $\omega$ set by the trap frequency, which also corresponds to the separation between the many multiplicities $(n)$ associated with the Floquet spectrum (since $\omega_{D}=\omega$ ); see Fig. 10 in Appendix C. Furthermore, as we have discussed, the junction regions that connect the reservoirs to the inner system (Fig. 5) can be thought of as uncoupled harmonic oscillator levels, leading to an effective multiterminal geometry where each row of sites corresponding to a given $\lambda$ is connected to two individual (fictitious) reservoirs ( $L_{\lambda}$ on the left and $R_{\lambda}$ on the right); see Sec. III B 2 and Fig. 7(c). In this picture, calculating the contribution of the $n$th mode to the conductance $G\left(\varepsilon_{F}+n \omega\right)$ is related to selecting the effective "terminals" $L_{n}$ and $R_{n}$ that are energetically resonant with the mode, namely, the terminals that are connected to the sites $\lambda=n$. In this sense, scanning through the Fourier modes is reminiscent of analyzing various transport channels in the effective multiterminal geometry [see Fig. 7(c)]: In particular, the contribution of the edge modes localized at $\lambda=0$ can be identified through the conductance $G_{L_{0}, R_{0}}$ associated with the lower terminals. In practice, measuring the contribution of a given Fourier mode $G_{L_{n}, R_{n}}$ can be achieved by setting the Fermi energy on resonance with respect to the corresponding harmonic oscillator states $(\lambda=n)$; see also Appendix C.

These observations lead to a remarkable corollary: Restricting the Floquet sum rule to a limited number of modes (set by $n_{\text {cut }}$ ) can be used as a method to isolate the contribution of individual chiral edge modes.


FIG. 9. Two-terminal conductance of the complete shakenchannel model defined in Eq. (8), as obtained from the Floquet RGF method. Parameters are $\lambda_{\text {cut }}=11, N_{y}=12, \varphi=2 \pi / 3$, $J_{y}=4 J_{1}, \kappa=0.03 \omega / l_{H}$, and $n_{F}=11$. (a) The conductance is obtained from the complete Floquet sum rule in Eq. (11); (b) individual contributions to the conductance corresponding to $n=0$ (blue), $n=1$ (orange), and $n=2$ (green). The main contribution to the plateau at $E_{F}>0$ stems from the $n=0$ component.

This particular feature of our synthetic dimension system allows for the unambiguous detection of the quantized Hall conductance in a two-reservoir setting. We further illustrate this important result in Sec. IV B based on the complete time-dependent model (Fig. 9).

As a technical remark, we note that the Floquet sum rule can be restricted to positive modes $(n \geq 0)$ in the present context, as the transmissions $T\left(\varepsilon_{F}+n \omega\right)$ associated with negative $n$ 's are found to have negligible contributions.

\section{B. Full-time-dependent model}

We finally discuss the transport properties of the fulltime-dependent model in Eq. (8), without neglecting the anisotropic hopping along the synthetic dimension $\lambda$. Here, we choose the hopping parameters $J_{y}=4 J_{1}$, such that the band gaps remain well open for $\lambda<30$; see Sec. III C.

We show the corresponding two-terminal conductance in Fig. 9(a), which is calculated using the complete Floquet sum rule (in the wideband limit). This result reproduces the quantized plateaus of Fig. 7, which are obtained using the effective-Hamiltonian approach. We note that the plateaus are slightly larger in Fig. 9(a), which is due to the fact that the band gap is slightly larger in the present configuration where $\lambda_{\text {cut }}=11$ and $N_{y}=12$.

In Fig. 9(b), we show individual contributions to the total conductance $G\left(\varepsilon_{F}+n \omega\right)$, considering the first three Fourier modes $n=0,1,2$; these are the first three nonvanishing contributions to the Floquet sum rule used in Fig. 9(a). As we discuss in Sec. IV A, the main contribution to the plateau at $E_{F}>0$ is due to the edge mode localized at $\lambda=0$, which is selected by the lowest $n \simeq 0$ Fourier component entering the sum rule. As can be seen in Fig. 9(b), the contribution of the $n=1$ component to the edge-mode signal is already substantially reduced, which is due to the highly localized nature of the edge mode. Similarly, we verify that the main contribution to 
the plateau at $\varepsilon_{F}<0$ mainly comes from the component $n_{F}=\lambda_{\text {cut }}$.

The result shown in Fig. 9(b) demonstrates how the quantized conductance associated with a topological edge mode (here, at $\lambda=0$ ) can be unambiguously detected using a few conductance measurements in a single atomic wire.

\section{EXPERIMENTAL CONSIDERATIONS}

We now discuss the experimental implementation of the shaken-channel scheme based on the demonstrated twoterminal cold-atom setup of Refs. [39,40]. As shown in Fig. 1, the quantum wire consists of a region with a tight harmonic confinement along both $x$ and $z$ directions. The propagation of atoms from one reservoir to the other in the $y$ direction is ballistic $[29,39,40]$. We consider, in line with Refs. [39,40], that the temperature is low enough such that individual harmonic oscillator states in the wire can be resolved by transport, yielding a quantized conductance upon varying the chemical potential (see Sec. IV B).

Using standard high-resolution optical techniques, the wire can be exposed to a periodic drive, while keeping the adiabatic connection to nonshaken reservoirs. The position-dependent phase of the temporal modulation in Eq. (4) can be realized using Raman transitions between harmonic oscillator states along one transverse direction. Alternatively, a direct time- and space-periodic deformation of the wire structure could be engineered based on lightshaping techniques. The typical length that can be achieved in these quantum wires is from 10 to $20(\mu \mathrm{m})$. The spatial period $l_{B}=2 \pi / \varphi$ associated with the time-dependent potential in Eq. (4) must be much shorter than the length of the wire itself. The driving strength $\kappa$ is controlled by the intensity of the Raman beams or the amplitude of the deformation and should satisfy the two following criteria: (i) ensure the validity of the rotating-wave approximation [56] for the relevant (low-energy) harmonic oscillator states participating to transport, which places an upper bound on the driving strength $\kappa$; (ii) create a topological bulk gap (hosting the edge modes) larger than temperature in order to be resolved by transport measurements, which constrains the strength from below. Based on the effective band gap of Fig. 7(a), we estimate the band gap to be $J_{y} \approx 2 \kappa \sqrt{1 / 2 M \omega}$, which has to fulfill $k_{B} T_{\text {temp }} \leq J_{y}$, with $T_{\text {temp }}$ the temperature of the atoms in the reservoirs. While these conditions are required for the above formalism to apply, we expect the physics to be robust against moderate deviations from these bounds. In addition, we note that realistic setups would typically involve 10-100 harmonic oscillator states in the channel, hence, offering a rather long synthetic dimension (as compared, for instance, to atomic-internal-states realizations [51,52]), and therefore, a good resolution of the edge-state signal.

As previously noted, there is no need for the projection of a lattice structure along the transport direction for the observation of the chiral edge states in the synthetic dimension, even though such a projection has recently been demonstrated [63]. Indeed, without a lattice the model maps onto the coupled-wires model of Refs. $[87,88]$ known to exhibit the quantum Hall effect.

The natural observable in the experiment is the twoterminal conductance measured as a function of the chemical potential. By repeating measurements for chemical potentials increased by an integer multiple of $\omega$, one can reconstruct the full conductance spectrum of the inner system, as indicated by the Floquet sum rule. A different type of measurement could be performed by making use of the direct observation of energy currents in two-terminal systems, as demonstrated in Refs. [92,93]. Indeed, even without resolving the topological band structure, a chemical-potential bias between the reservoirs will yield a current in the $\lambda$ direction, which will contribute to the thermopower of the channel and provide a direct measure of the chirality of the underlying model.

\section{CONCLUSIONS}

We propose a scheme by which the quantum Hall conductance of a neutral atomic gas can be detected using a minimal one-dimensional setting: a quantum wire connected to two reservoirs [39,40]. The twodimensional nature of the quantum Hall effect is offered by an additional (synthetic) dimension, which is naturally present in the system. Inspired by Ref. [56], we propose that a Chern-insulating state (realizing the quantum Hall effect) can be realized in this setting upon subjecting the quantum wire to a resonant modulation. Importantly, the resulting quantized Hall conductance can be unambiguously detected in this scheme by exploiting an unusual feature offered by the synthetic dimension: Its energetic nature effectively leads to a multiterminal geometry, which allows for a clear measurement of the chiral edge modes' contribution to transport. This appealing result is demonstrated using two complementary approaches, one based on effective (time-independent) Hamiltonians and the other on a Floquet-Landauer approach, which takes the full time dependence of the problem into account.

Intriguing perspectives include the study of interparticle interactions in this synthetic dimension approach. As discussed in Ref. [56], interactions are long-ranged (but not infinite range) along the synthetic dimension, and the corresponding phases are still to be elucidated. In particular, it would be interesting to identify regimes where strongly correlated states with topological features could be stabilized in this setting; the corresponding Hall conductance could then be explored using the schemes and concepts detailed in the present work.

The notions and results introduced in this work could be applied to other physical platforms. For instance, synthetic dimensions have been proposed in the context of photonics 
$[59,62,94-96]$, and a first experimental realizationreminiscent of the scheme proposed in Ref. [56]—was recently reported in Ref. [61]. In this context, we note that a transport formalism (analogous to the Landauer formalism) has been proposed to describe transport of photons [97]. Altogether, these advances suggest that the scheme discussed in this work could be directly transposed to the context of topological photonics [98].

Finally, this work emphasizes the richness and complexity of quantum transport in the general context of Floquetengineered systems. As is highlighted in our study (and as particularly emphasized in Appendix C), a conventional configuration of the reservoirs (which creates a weak chemical-potential imbalance on either side of the system under scrutiny) leads to a intricate (nonuniform) occupation of the Floquet eigenstates associated with the inner system. While this generically complicates the analysis of such driven settings, we show how to take advantage of this unusual feature in order to finely probe and identify the transport properties of topological edge modes. We believe that such an interplay between quantum transport, Floquet engineering, and topological edge modes will play a crucial role in near-future experiments.

\section{ACKNOWLEDGMENTS}

We acknowledge the KWANT code [99], which is used to benchmark some of the results shown in this paper. We are grateful to Tomoki Ozawa, Alexandre Dauphin, and Marco Di Liberto for fruitful discussions, and Philipp Fabritius for careful reading of the manuscript. Work in Brussels is supported by the Fonds De La Recherche Scientifique (FRS-FNRS, Belgium) and the ERC Starting Grant TopoCold. H. M. P. is supported by funding from the Royal Society via Grants No. UF160112, No. RGFIEA\180121, and No. RGF \R1\180071. J.-P. B. is supported by the ERC project DECCA (Project No. 714309) and the Sandoz Family FoundationMonique de Meuron program for Academic Promotion. L. C. is supported by ETH Zurich Postdoctoral Fellowship and Marie Curie Actions for People COFUND program and ERC Marie Curie TopSpiD (Project No. 746150). M. L., S. H., and T.E.'s work is supported by ERC advanced grant TransQ (Project No. 742579).

\section{APPENDIX A: LANDAUER-BÜTTIKER FORMALISM AND NONEQUILIBRIUM GREEN'S FUNCTION}

In this Appendix, we review the theoretical framework offered by the Landauer-Büttiker formalism, which was originally developed for calculating the conductance in solid-state systems $[5,6,25,26]$, but which has also been recently applied to describe transport in charge-neutral atomic systems [29]. We first consider the case of a single channel connected to two external reservoirs, which act as contact terminals and are labeled by $\alpha$ and $\beta$; throughout, we assume that particles obey Fermi statistics. The chemical potential in the $\alpha$ (resp. $\beta$ ) reservoir is denoted $\mu_{\alpha}$ (resp. $\left.\mu_{\beta}\right)$; we assume that these chemical potentials are centered around the Fermi energy $E_{F}$ and that their differences are small. In this case, and assuming zero temperature for now, the linear dc current that flows between the two reservoirs can be expressed as

$$
I_{\alpha, \beta}=G_{\alpha, \beta}\left(E_{F}\right)\left(\mu_{\alpha}-\mu_{\beta}\right),
$$

where $G_{\alpha, \beta}\left(E_{F}\right)$ is the conductance of the system at a given Fermi energy $E_{F}$ [25]. This conductance can be evaluated using the Landauer formula

$$
G_{\alpha, \beta}\left(E_{F}\right) \equiv \frac{1}{h} \sum_{m} T_{\alpha, \beta}^{(m)}\left(E_{F}\right),
$$

which involves a sum over the $m$ possible transport channels of the transmission probabilities $T_{\alpha, \beta}^{(m)}\left(E_{F}\right)$ for a particle of charge $e$ to be carried through the system. The transmission probabilities are related to the scattering properties of the system [5]; in particular, if the system has $N$ perfectly transmitting channels, i.e., $T^{(m)}=1$ for all $m$, each of these will contribute with a quantum of conductance $G_{0}=1 / h$ such that the total conductance is quantized according to $G_{\alpha, \beta}=N G_{0}$.

For a system connected to many reservoirs, the dc current at a given terminal $\alpha$ is generalized by the Landauer-Büttiker formula

$$
I_{\alpha}=\sum_{\beta} I_{\alpha, \beta}=\sum_{\beta} G_{\alpha, \beta}\left(E_{F}\right)\left(\mu_{\alpha}-\mu_{\beta}\right),
$$

where the sum is now taken over all other reservoirs $\beta$, which are connected to $\alpha$.

In the case of finite temperature, the currents in Eqs. (A1) and (A3) must be weighted with the Fermi-Dirac distributions evaluated at the two reservoirs $f_{\alpha} \equiv f\left(E-\mu_{\alpha}\right)$ and $f_{\beta} \equiv f\left(E-\mu_{\beta}\right)$ and then integrated over all energies, which yields the following generalized expression [100]:

$$
I_{\alpha} \equiv \frac{1}{h} \sum_{\beta, m} \int T_{\alpha, \beta}^{(m)}(E)\left[f_{\alpha}-f_{\beta}\right] d E .
$$

To evaluate the transmission probabilities in Eq. (A2), it is often convenient to use the nonequilibrium Green's function method, which is mathematically equivalent to the scattering approach in the linear regime [26,80]. The matrix representation of the retarded Green's function $\mathcal{G}^{r}$ of a system at Fermi energy $E_{F}$ is defined through the Hamiltonian matrix $\mathbf{H}$ as

$$
[\mathbf{E}-\mathbf{H}] \mathcal{G}^{r}=\mathbb{I},
$$


where $\mathbf{E}=\left(E_{F}+i 0^{+}\right) \mathbb{I}, \mathbb{I}$ is the identity matrix, and $0^{+}$is an infinitesimally small positive quantity.

Without loss of generality, we focus on a channel connected to two reservoirs, which are attached to the left and the right of the system (in this setting, $\alpha, \beta=R, L$ refer to the two reservoirs). The Hamiltonian matrix of the entire scattering system, including the reservoirs, has the following block structure

$$
\mathbf{H}_{\mathrm{tot}}=\left(\begin{array}{ccc}
\mathbf{H}_{L} & \mathbf{H}_{L S} & 0 \\
\mathbf{H}_{L S}^{\dagger} & \mathbf{H}_{S} & \mathbf{H}_{R S} \\
0 & \mathbf{H}_{R S}^{\dagger} & \mathbf{H}_{R}
\end{array}\right),
$$

where $\mathbf{H}_{L, R}$ refers to the Hamiltonians describing the left or right reservoirs, $\mathbf{H}_{S}$ describes the inner system (the transport channel), and where $\mathbf{H}_{L S}$ and $\mathbf{H}_{R S}$ describe the couplings between the inner system and the left or right reservoirs. Since the size of the reservoirs is typically very large, the size of the Hamiltonian matrices $\mathbf{H}_{L, R}$ is large compared to the size of $\mathbf{H}_{S}$. From Eq. (A5), the Green's function of the total system is

$$
\begin{gathered}
\left(\begin{array}{ccc}
\mathbf{E}-\mathbf{H}_{L} & -\mathbf{H}_{L S} & 0 \\
-\mathbf{H}_{L S}^{\dagger} & \mathbf{E}-\mathbf{H}_{S} & -\mathbf{H}_{R S} \\
0 & -\mathbf{H}_{R S}^{\dagger} & \mathbf{E}-\mathbf{H}_{R}
\end{array}\right)^{-1} \\
=\left(\begin{array}{ccc}
\mathcal{G}_{L}^{r} & \mathcal{G}_{L S}^{r} & 0 \\
\mathcal{G}_{L S}^{r} & \mathcal{G}_{S}^{r} & \mathcal{G}_{R S}^{r} \\
0 & \mathcal{G}_{R S}^{r}{ }^{\dagger} & \mathcal{G}_{R}^{r}
\end{array}\right) .
\end{gathered}
$$

From Eq. (A7), one can obtain the following relation for the Green's function of the inner system $\mathcal{G}_{S}^{r}$,

$$
\left\{\mathbf{E}-\mathbf{H}_{S}-\left[\boldsymbol{\Sigma}_{L}\left(E_{F}\right)+\boldsymbol{\Sigma}_{R}\left(E_{F}\right)\right]\right\} \mathcal{G}_{S}^{r}=\mathbb{I}
$$

where $\boldsymbol{\Sigma}_{L}\left(E_{F}\right)=\mathbf{H}_{L S}^{\dagger}\left(\mathbf{E}-\mathbf{H}_{L}\right)^{-1} \mathbf{H}_{L S}$ and $\boldsymbol{\Sigma}_{R}\left(E_{F}\right)=$ $\mathbf{H}_{R S}\left(\mathbf{E}-\mathbf{H}_{R}\right)^{-1} \mathbf{H}_{R S}^{\dagger}$. Equation (A8) is very similar to Eq. (A5), except that the Hamiltonian is now modified with the term $\boldsymbol{\Sigma}\left(E_{F}\right)=\sum_{\alpha} \boldsymbol{\Sigma}_{\alpha}\left(E_{F}\right)$, the so-called contact self-energy, which includes the details of the reservoirs. The anti-Hermitian counterpart of the self-energy $\boldsymbol{\Gamma}^{\alpha}\left(E_{F}\right)=$ $i\left(\boldsymbol{\Sigma}_{\alpha}\left(E_{F}\right)-\boldsymbol{\Sigma}_{\alpha}^{\dagger}\left(E_{F}\right)\right)$ defines the linewidth of the $\alpha$ reservoir, and it reflects the fact that particles are lost from the inner system due to leakage into the reservoirs; in this sense, the channel is out of equilibrium [26]. The linewidth of the reservoir and the self-energy contributions can be obtained following standard prescriptions [26,81].

Using the nonequilibrium Keldysh formalism [101], the transmission can be calculated through the Caroli formula [82], which involves the Green's functions of the inner system and the self-energies of the reservoirs:

$$
T_{\alpha, \beta}\left(E_{F}\right)=\operatorname{Tr}\left[\boldsymbol{\Gamma}^{\alpha}\left(E_{F}\right) \mathcal{G}^{r}\left(E_{F}\right) \boldsymbol{\Gamma}^{\beta}\left(E_{F}\right) \mathcal{G}^{a}\left(E_{F}\right)\right],
$$

where we omit the subscript $S$ associated with the Green's functions of the system for simplicity of notation; we note that the advanced Green's function satisfies $\mathcal{G}^{a}=\left(\mathcal{G}^{r}\right)^{\dagger}$. Equation (A9) is very convenient for numerical evaluations of the dc current that flows between two terminals when combined with the RGF method [81,83] based on the Dyson equation. The RGF method can be generalized to multiterminal systems [84], which we use for calculating the Hall conductance later in this article.

\section{The wideband approximation}

We see that all the details of the reservoirs are included in the self-energy matrix $\boldsymbol{\Sigma}\left(E_{F}\right)$, which is used both for obtaining the linewidth $\boldsymbol{\Gamma}\left(E_{F}\right)$ and the Green's function of the inner system connected to the terminals. In order to calculate the self-energy $\boldsymbol{\Sigma}\left(E_{F}\right)$, the reservoir is typically assumed to have a large volume and a high density of states. If the density of states of the reservoir is constant over an energy range much larger that the bandwidth of the inner system, the wideband approximation can be used [102]. Under this approximation, both the self-energy and the linewidth are taken to be energy independent. In particular $\boldsymbol{\Sigma}_{\alpha} \propto-i \mathbb{I} \delta_{x, x_{\alpha}} \gamma$, where $\gamma$ is a constant that is of the order of the bandwidth of the inner system, and the $\delta_{x, x_{\alpha}}$ selects only the sites of the system that belong to the terminal $\alpha$ [75]. Unless otherwise stated, the wideband approximation is used for all the results presented in the main text.

\section{APPENDIX B: EVALUATING THE CONDUCTANCE IN PERIODICALLY DRIVEN SYSTEMS: THE FLOQUET-LANDAUER APPROACH}

As we see, our proposal builds on the possibility of addressing a synthetic dimension by applying a timeperiodic modulation to an atomic channel [56]. In fact, this proposed scheme belongs to the general class of Floquet-engineered quantum systems, which aim to realize intriguing Hamiltonian models through periodic driving [70,72,103-107]. In this context, it is common to derive an effective (Floquet) Hamiltonian that describes the longtime dynamics of the system and which results from a rich interplay between the time modulation and the underlying static system [70,72]. In fact, Floquet engineering can also be exploited to transfigure quantum-transport properties, in the sense that applying a temporal modulation can greatly modify the transport channels of a quantum system. In this quantum-transport framework, where the time-modulated system is further connected to reservoirs, it is generally insufficient to simply apply the standard tools of quantumtransport theory to the effective (Floquet) Hamiltonian, which describes the inner system; such a naive approach is discussed in Sec. III. Instead, a more rigorous approach consists of using a generalization of the non-equilibrium Green's function method [73-75,77], which is specifically 
tailored to treat time-periodic systems, as we now review in this Appendix; this approach is applied in Sec. IV.

Consider a time-dependent Hamiltonian $\mathbf{H}(t+T)=$ $\mathbf{H}(t)$, where $T=2 \pi / \omega$ is the period of the applied temporal modulation. The Fourier expansion of the Hamiltonian yields

$$
\mathbf{H}(t)=\sum_{n=-n_{F}}^{n_{F}} \mathbf{H}_{n} e^{-i n \omega t},
$$

where we truncate the series up to $N_{F}=2 n_{F}+1$ modes. In Floquet systems, the energy is defined only up to multiples of the driving frequency $E=\varepsilon+n \omega$ (hereafter, we set $\hbar=1$ ). This observation leads to the notion of quasienergies $\varepsilon$, which can be chosen within the Brillouin zone $\varepsilon \in[-\omega / 2, \omega / 2]$; see the review [72]. The so-called Floquet spectrum, which is defined in this restricted range, is then periodically repeated for each multiplicity (i.e., around each $n \omega$ ). As discussed in Ref. [90], it is convenient to treat such periodically driven systems in an extended (Floquet) Hilbert space of dimension $N_{x} N_{y} N_{F}$, which explicitly takes these multiplicities into account. In this framework, the Hamiltonian is replaced by a so-called "quasienergy operator" $\mathbf{Q}$, whose components $Q_{m, n}=$ $\mathbf{H}_{m-n}+\delta_{m, n} m \omega \mathbb{I}$ act in the original Hilbert space; here, $\mathbf{H}_{n}$ refers to the Fourier components introduced in Eq. (B1), and $\mathbb{I}$ is the $N_{x} \times N_{y}$ identity matrix.

Following Refs. [74,75], one generalizes Eq. (A8) in view of defining a Floquet representation for the Green's function through the relation

$$
\left[\mathbf{E}-\mathbf{Q}-\left(\boldsymbol{\Sigma}_{F}^{R}+\boldsymbol{\Sigma}_{F}^{L}\right)\right] \mathcal{G}_{F}^{r}=\mathcal{I},
$$

where all matrices are defined in the extended Floquet Hilbert space of dimension $N_{x} N_{y} N_{F}$, and where $\mathcal{I}$ is the identity matrix in this extended space. Specifically, $\mathbf{E}$ and $\boldsymbol{\Sigma}_{F}$ are diagonal matrices whose elements are

$$
\begin{array}{r}
\mathbf{E} \equiv \operatorname{Diag}\left[\left(E_{F}+i 0^{+}\right) \mathbb{I}, \ldots,\left(E_{F}+i 0^{+}\right) \mathbb{I}\right], \\
\boldsymbol{\Sigma}_{F}^{\alpha} \equiv \operatorname{Diag}\left[\boldsymbol{\Sigma}_{\alpha}\left(E_{F}-n_{F} \omega\right), \ldots, \boldsymbol{\Sigma}_{\alpha}\left(E_{F}+n_{F} \omega\right)\right],
\end{array}
$$

where $\alpha=L, R$ and $\boldsymbol{\Sigma}_{\alpha}\left(E_{F}\right)$ is defined below Eq. (A8). Solving Eq. (B2) yields the Floquet representation for the Green's function, $\mathcal{G}_{F}^{r}$ :

$$
\mathcal{G}_{F}^{r} \equiv\left(\begin{array}{ccccc}
\mathcal{G}_{-n_{F},-n_{F}}^{r} & \ldots & \mathcal{G}_{-n_{F}, 0}^{r} & \ldots & \mathcal{G}_{-n_{F}, n_{F}}^{r} \\
\vdots & \ddots & \vdots & \ldots & \vdots \\
\mathcal{G}_{0,-n_{F}}^{r} & \ldots & \mathcal{G}_{0,0}^{r} & \ldots & \mathcal{G}_{0, n_{F}}^{r} \\
\vdots & \ldots & \vdots & \ddots & \vdots \\
\mathcal{G}_{n_{F},-n_{F}}^{r} & \ldots & \mathcal{G}_{n_{F}, 0}^{r} & \ldots & \mathcal{G}_{n_{F}, n_{F}}^{r}
\end{array}\right)
$$

As in Appendix A, the linewidth can be defined as $\boldsymbol{\Gamma}^{\alpha}=i\left(\boldsymbol{\Sigma}_{\alpha F}-\boldsymbol{\Sigma}_{\alpha F}^{\dagger}\right)$, and it can also be represented in the extended Hilbert space (with components denoted $\boldsymbol{\Gamma}_{n, m}^{\alpha}$ ).

The Floquet generalization of the Caroli formula in Eq. (A9) can then be obtained by treating the Fourier components of the Green's function and the linewidth individually. The resulting Floquet-Caroli formula for the transmission at a given Fermi energy $E_{F}$ reads [75]

$T_{\alpha, \beta}\left(E_{F}\right)=\sum_{n=-n_{F}}^{n_{F}} \operatorname{Tr}\left[\boldsymbol{\Gamma}_{0,0}^{\alpha}\left(E_{F}\right) \mathcal{G}_{n, 0}^{r}\left(E_{F}\right) \boldsymbol{\Gamma}_{n, n}^{\beta}\left(E_{F}\right) \mathcal{G}_{0, n}^{a}\left(E_{F}\right)\right]$,

where $\boldsymbol{\Gamma}_{n, m}^{\alpha}$ denote the components of the linewidth $\boldsymbol{\Gamma}^{\alpha}$ introduced above.

\section{Floquet sum rule}

In the framework of periodically driven systems, one is interested in studying the contribution of quasienergy bands $\varepsilon(\mathbf{k})$ to the conductance. In particular, if a quasienergy band is associated with a nonzero Chern number, one would expect to observe a quantized Hall conductance, in direct analogy with the static case. However, this analysis requires special care, as previously highlighted in Refs. [74,75,91].

In order to draw an analogy with static systems, one introduces a Fermi quasienergy $\varepsilon_{F}$, which is defined as $E_{F}=\varepsilon_{F}+n \omega$, where $E_{F}$ is the Fermi energy set by the static reservoirs, and where $\varepsilon_{F} \in[-\omega / 2, \omega / 2]$ scans the quasienergy spectrum within a single Floquet-Brillouin zone. Interpreting the quasienergy spectrum as the energy spectrum associated with a static system, one is interested in evaluating the conductance at a given $\varepsilon_{F}$ (i.e., interpreting $\varepsilon_{F}$ as the standard Fermi energy). As previously shown in Refs. [75,91], this conductance calculation can be achieved by summing over the transmissions associated with all multiplicities [75,91]:

$$
T_{\alpha, \beta}\left(\varepsilon_{F}\right)=\sum_{n \in \mathbb{Z}} T_{\alpha, \beta}\left(\varepsilon_{F}+n \omega\right) .
$$

As we illustrate in the main text, the sum rule in Eq. (B7) is essential to recover a quantized Hall conductance in periodically driven systems realizing the quantum Hall effect (and Floquet Chern insulators in general [74]). The transmission is calculated from Eq. (B6) together with Eq. (B7) as

$$
\begin{aligned}
T_{\alpha, \beta}\left(\varepsilon_{F}\right)= & \sum_{n, n^{\prime}=-n_{F}, \ldots, n_{F}} \operatorname{Tr}\left[\boldsymbol{\Gamma}_{0,0}^{\alpha}\left(\varepsilon_{F}+n \omega\right)\right. \\
& \left.\times \mathcal{G}_{n^{\prime}, 0}^{r}\left(\varepsilon_{F}+n \omega\right) \boldsymbol{\Gamma}_{n^{\prime}, n^{\prime}}^{\beta}\left(\varepsilon_{F}+n \omega\right) \mathcal{G}_{0, n^{\prime}}^{a}\left(\varepsilon_{F}+n \omega\right)\right] .
\end{aligned}
$$


The sum rule in Eq. (B7) also leads to an interesting experimental corollary, which is that the conductance cannot be evaluated based on a single measurement. As discussed in Ref. [75], the transport experiment should be repeated for various values of the reservoirs' chemical potential, which should be chosen so as to probe the many multiplicities $\varepsilon_{F}+n \omega$. The convergence of this approach is illustrated in Sec. IV.

\section{APPENDIX C: THE FLOQUET EIGENSTATES IN THE CHANNEL AND THE BARE LEVELS IN THE STATIC RESERVOIRS}

This Appendix aims to further deepen our understanding of the Floquet system studied in Sec. IV by analyzing how the states of the static system (i.e., the reservoir and junction regions) project onto the states of the shaken system (inner region).

We start by displaying in Fig. 10 a schematic picture of the energy levels in the channel, where the color scale highlights the low- $\lambda$ levels with darker colors. In the

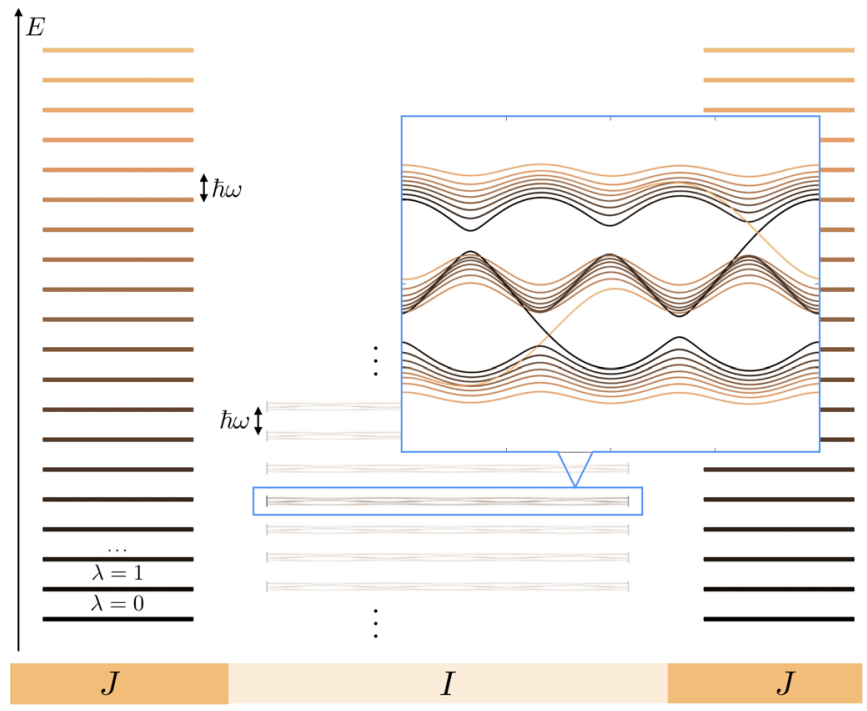

FIG. 10. Schematic representation of the energy levels of the junction regions $(J)$ and the inner region $(I)$. The color scale is chosen as in Fig. 7(a) and indicates the mean position $\langle\lambda\rangle$ of the states. The levels in the junction region are the harmonic oscillator states separated by $\lambda \omega$. The quasienergy spectrum $\varepsilon\left(k_{y}\right)$ of the inner region contains contributions from all the $\lambda$ states due to the coupling provided by the shaking. Here the Floquet spectrum is represented in an extended zone scheme, according to which the same Floquet eigenstates are repeated periodically, $E=\varepsilon+n \omega$. We note that the separation between these Floquet multiplicities matches the separation between the bare $\lambda$ states. The dispersion of the states in the junction due to propagation along the $y$ direction is associated with a small bandwidth $J_{y} \ll \hbar \omega$, which is of the same order as the bandwidth of the quasienergy spectrum in the channel. The population of the Floquet eigenstates within the channel is nonthermal but reflects the thermal population in the undriven reservoirs (see text). junction regions, the energy levels are the equally spaced harmonic oscillator states labeled by $\lambda$. Within the inner region, the driving protocol couples all the $\lambda$ states together, and the quasienergy dispersion $\varepsilon$ corresponds to that of the effective model in Eq. (6), whose bandwidth is of order $J_{y}$. Because of the driven nature of the inner system, this quasienergy spectrum can be repeated periodically $E=\varepsilon+n \omega$, where $n$ labels the many replicates. As indicated in Fig. 10, the spacing between these multiplicities also corresponds to the energy separation between the bare $\lambda$ states in the junction regions; it should also be noted that the states in the junction regions are associated with a finite dispersion due to the motion along the $y$ direction, which is characterized by a bandwidth of order $J_{y}$.

We now address the following question: Considering that the Fermi energy $E_{F}$ set in the reservoirs is such that only the first few low- $\lambda$ states are populated, what are the (Floquet) eigenstates of the inner region that are predominantly occupied and hence contribute to transport? Importantly, we stress that the population of the different energy levels within the channel is nonthermal but reflects the thermal population of the uncoupled $\lambda$ states in the reservoirs. Although very schematic, we already observe from Fig. 10 that the low- $\lambda$ states in the junction regions (black) mainly overlap with the midgap edge states of the inner (shaken) region, with only small overlaps with bulk states; hence, we expect these edge states to be significantly populated in this reservoir configuration.

To further illustrate this point, we plot in Fig. 11 the energy spectrum of the effective time-independent model and highlight those states that are strongly localized around $\lambda=0,1,2,3$, respectively (we recall that this model is defined in the $\lambda-y$ plane, where $\lambda$ refers to the synthetic dimension coordinate). We also plot the corresponding amplitudes $|\psi(\lambda)|^{2}$ for the special states indicated by black dots. We note that the midgap edge state (i.e., the $B$ state in Fig. 11) is indeed sharply localized along $\lambda=0$ and that the bare states on the next row $(\lambda=1)$ already have a very little overlap with it. From this very simple "decomposition" of the inner-system quasienergy spectrum [see also Fig. 7(a) of the main text], we deduce that a chiral edge transport occurs along the channel (i.e., along the $\lambda=0$ axis of the hybrid 2D system) whenever the Fermi energy is set in the vicinity of the lowest harmonic oscillator state $\lambda$ in the reservoirs. This understanding is compatible with the result shown in Fig. 9(b), where the main contribution to the plateau in the conductance spectrum (at positive energy) is shown to result from the $n=0$ Fourier component, namely, when the Fermi energy $E_{F}=\varepsilon_{F}+n \omega$ is set around the $\lambda=0$ state of the harmonic oscillator.

It is important to notice that although the reservoir imposes a standard (thermal) Fermi distribution to the junction region, the bare $\lambda$ states project very differently on the various states of the Floquet inner system, hence, giving rise to a nonstandard (nonthermal) distribution of 

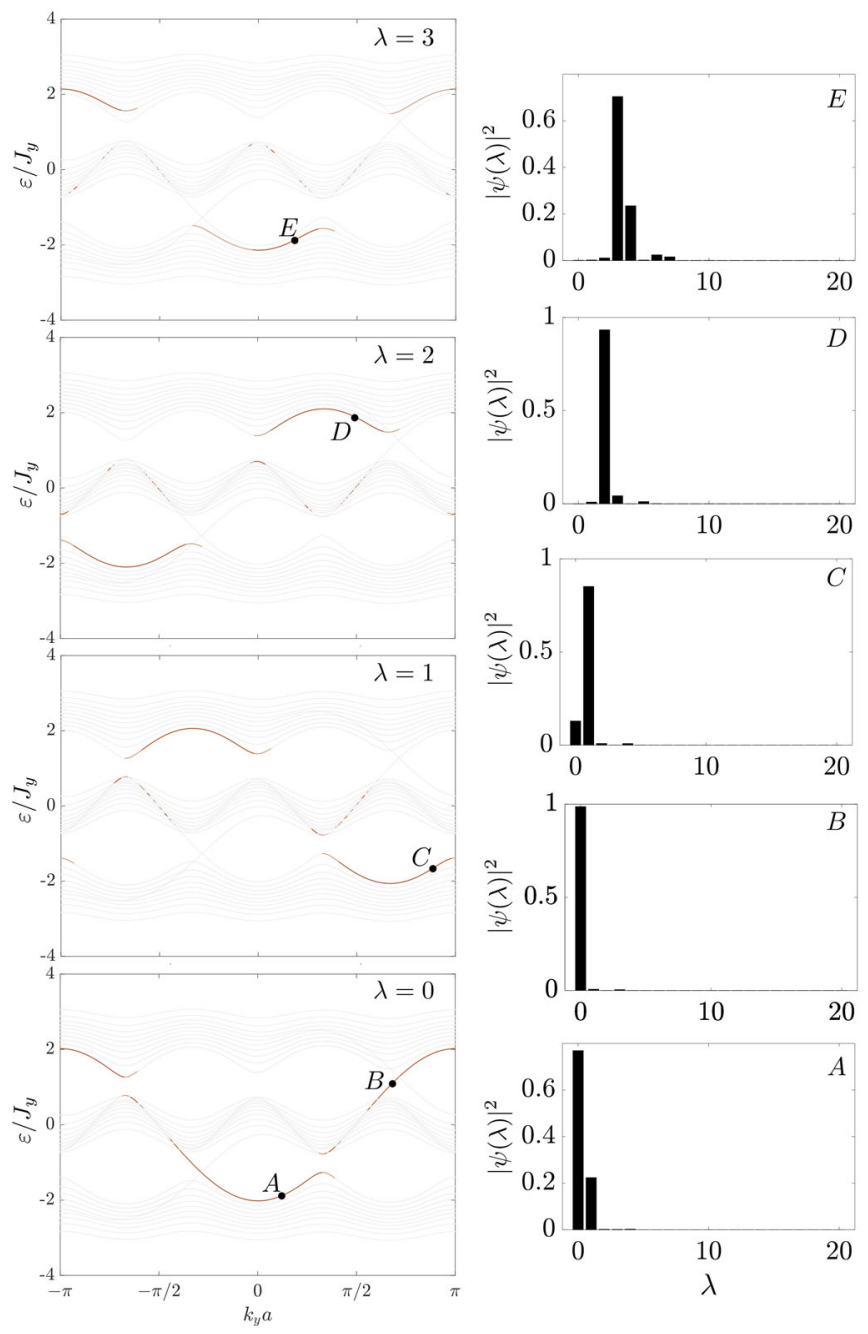

FIG. 11. Energy spectra as in Fig. 7(a), where we show only the parts of the spectra whose states are localized around $\lambda=0,1,2$, 3 , respectively. The amplitudes $|\psi(\lambda)|^{2}$ of the states indicated with a dot are also shown.

populations. In fact, the state occupation depends also on the localization along the synthetic dimension, and that is a crucial difference with the usual Fermi distribution: Setting a given Fermi energy $E_{F}=\varepsilon_{F}+n \omega$ in the reservoir does not imply that all the states in the inner system are empty if they lie above $\varepsilon_{F}$ or that they are occupied if they are below $\varepsilon_{F}$.

[1] Y. Imry, Introduction to Mesoscopic Physics (Oxford University Press, New York, 1997).

[2] B. J. van Wees, H. van Houten, C. W. J. Beenakker, J. G. Williamson, L. P. Kouwenhoven, D. van der Marel, and C. T. Foxon, Quantized Conductance of Point Contacts in a Two-Dimensional Electron Gas, Phys. Rev. Lett. 60, 848 (1988).

[3] D. A. Wharam, T. J. Thornton, R. Newbury, M. Pepper, H. Ahmed, J. E. F. Frost, D. G. Hasko, D. C. Peacock,
D. A. Ritchie, and G. A.C. Jones, One-Dimensional Transport and the Quantisation of the Ballistic Resistance, J. Phys. C 21, L209 (1988).

[4] J. M. Krans, J. M. van Ruitenbeek, V. V. Fisun, I. K. Yanson, and L. J. de Jongh, The Signature of Conductance Quantization in Metallic Point Contacts, Nature (London) 375, 767 (1995).

[5] R. Landauer, Spatial Variation of Currents and Fields Due to Localized Scatterers in Metallic Conduction, IBM J. Res. Dev. 1, 223 (1957).

[6] M. Büttiker, Y. Imry, R. Landauer, and S. Pinhas, Generalized Many-Channel Conductance Formula with Application to Small Rings, Phys. Rev. B 31, 6207 (1985).

[7] K. von Klitzing, G. Dorda, and M. Pepper, New Method for High-Accuracy Determination of the Fine-Structure Constant Based on Quantized Hall Resistance, Phys. Rev. Lett. 45, 494 (1980).

[8] M. O. Goerbig, in Quantum Hall Effects, edited by C. Miniatura, L.-C. Kwek, M. Ducloy, B. Grémaud, B.-G. Englert, L. Cugliandolo, A. Ekert, and K. K. Phua, Proceedings of the Les Houches Summer School (Oxford University Press, New York, 2009).

[9] D. Yoshioka, The Quantum Hall Effect (Springer, Berlin, 2002).

[10] D. J. Thouless, M. Kohmoto, M. P. Nightingale, and M. den Nijs, Quantized Hall Conductance in a Two-Dimensional Periodic Potential, Phys. Rev. Lett. 49, 405 (1982).

[11] Q. Niu, D. J. Thouless, and Y.-S. Wu, Quantized Hall Conductance as a Topological Invariant, Phys. Rev. B 31, 3372 (1985).

[12] R. B. Laughlin, Quantized Hall Conductivity in Two Dimensions, Phys. Rev. B 23, 5632 (1981).

[13] B. I. Halperin, Quantized Hall Conductance, CurrentCarrying Edge States, and the Existence of Extended States in a Two-Dimensional Disordered Potential, Phys. Rev. B 25, 2185 (1982).

[14] A. H. MacDonald, Edge States and Quantized Hall Conductivity in a Periodic Potential, Phys. Rev. B 29, 6563 (1984).

[15] Y. Hatsugai, Chern Number and Edge States in the Integer Quantum Hall Effect, Phys. Rev. Lett. 71, 3697 (1993).

[16] D. C. Tsui, H. L. Stormer, and A. C. Gossard, TwoDimensional Magnetotransport in the Extreme Quantum Limit, Phys. Rev. Lett. 48, 1559 (1982).

[17] R. B. Laughlin, Anomalous Quantum Hall Effect: An Incompressible Quantum Fluid with Fractionally Charged Excitations, Phys. Rev. Lett. 50, 1395 (1983).

[18] F. D. M. Haldane, Fractional Quantization of the Hall Effect: A Hierarchy of Incompressible Quantum Fluid States, Phys. Rev. Lett. 51, 605 (1983).

[19] C. L. Kane and E. J. Mele, Quantum Spin Hall Effect in Graphene, Phys. Rev. Lett. 95, 226801 (2005).

[20] C. L. Kane and E. J. Mele, $Z_{2}$ Topological Order and the Quantum Spin Hall Effect, Phys. Rev. Lett. 95, 146802 (2005).

[21] B. A. Bernevig and S.-C. Zhang, Quantum Spin Hall Effect, Phys. Rev. Lett. 96, 106802 (2006).

[22] M. König, S. Wiedmann, C. Brüne, A. Roth, H. Buhmann, L. W. Molenkamp, X.-L. Qi, and S.-C. Zhang, Quantum 
Spin Hall Insulator State in HgTe Quantum Wells, Science 318, 766 (2007).

[23] P. Hosur and X. Qi, Recent Developments in Transport Phenomena in Weyl Semimetals, C.R. Phys. 14, 857 (2013).

[24] N. P. Armitage, E. J. Mele, and A. Vishwanath, Weyl and Dirac Semimetals in Three-Dimensional Solids, Rev. Mod. Phys. 90, 015001 (2018).

[25] Y. Imry and R. Landauer, Conductance Viewed as Transmission, Rev. Mod. Phys. 71, S306 (1999).

[26] S. Datta, Quantum Transport: Atom to Transistor (Cambridge University Press, Cambridge, England, 2005).

[27] L. Amico, G. Birkl, M. Boshier, and L.-C. Kwek, Focus on Atomtronics-Enabled Quantum Technologies, New J. Phys. 19, 020201 (2017).

[28] I. Bloch, J. Dalibard, and W. Zwerger, Many-Body Physics with Ultracold Gases, Rev. Mod. Phys. 80, 885 (2008).

[29] S. Krinner, T. Esslinger, and J.-P. Brantut, Two-Terminal Transport Measurements with Cold Atoms, J. Phys. Condens. Matter 29, 343003 (2017).

[30] U. Schneider, L. Hackermüller, J. P. Ronzheimer, S. Will, S. Braun, T. Best, I. Bloch, E. Demler, S. Mandt, D. Rasch et al., Fermionic Transport and Out-of-Equilibrium Dynamics in a Homogeneous Hubbard Model with Ultracold Atoms, Nat. Phys. 8, 213 (2012).

[31] S. Scherg, T. Kohlert, J. Herbrych, J. Stolpp, P. Bordia, U. Schneider, F. Heidrich-Meisner, I. Bloch, and M. Aidelsburger, Nonequilibrium Mass Transport in the 1D Fermi-Hubbard Model, Phys. Rev. Lett. 121, 130402 (2018).

[32] P. T. Brown, D. Mitra, E. Guardado-Sanchez, R. Nourafkan, A. Reymbaut, C.-D. Hébert, S. Bergeron, A. M. S. Tremblay, J. Kokalj, D. A. Huse, P. Schauß, and W. S. Bakr, Bad Metallic Transport in a Cold Atom Fermi-Hubbard System, Science 363, 379 (2019).

[33] M. C. Beeler, R. A. Williams, K. Jiménez-García, L. J. LeBlanc, A. R. Perry, and I. B. Spielman, The Spin Hall Effect in a Quantum Gas, Nature (London) 498, 201 (2013).

[34] M. B. Dahan, E. Peik, J. Reichel, Y. Castin, and C. Salomon, Bloch Oscillations of Atoms in an Optical Potential, Phys. Rev. Lett. 76, 4508 (1996).

[35] G. Jotzu, M. Messer, R. Desbuquois, M. Lebrat, T. Uehlinger, D. Greif, and T. Esslinger, Experimental Realization of the Topological Haldane Model with Ultracold Fermions, Nature (London) 515, 237 (2014).

[36] M. Aidelsburger, M. Lohse, C. Schweizer, M. Atala, J. T. Barreiro, S. Nascimbène, N. R. Cooper, I. Bloch, and N. Goldman, Measuring the Chern Number of Hofstadter Bands with Ultracold Bosonic Atoms, Nat. Phys. 11, 162 (2015).

[37] R. Anderson, F. Wang, P. Xu, V. Venu, S. Trotzky, F. Chevy, and J. H. Thywissen, Optical Conductivity of a Quantum Gas, Phys. Rev. Lett. 122, 153602 (2019).

[38] L. Asteria, D. T. Tran, T. Ozawa, M. Tarnowski, B. S. Rem, N. Fläschner, K. Sengstock, N. Goldman, and C. Weitenberg, Measuring Quantized Circular Dichroism in Ultracold Topological Matter, Nat. Phys. 15, 449 (2019).
[39] J.-P. Brantut, J. Meineke, D. Stadler, S. Krinner, and T. Esslinger, Conduction of Ultracold Fermions through a Mesoscopic Channel, Science 337, 1069 (2012).

[40] S. Krinner, D. Stadler, D. Husmann, J.-P. Brantut, and T. Esslinger, Observation of Quantized Conductance in Neutral Matter, Nature (London) 517, 64 (2015).

[41] D. Husmann, S. Uchino, S. Krinner, M. Lebrat, T. Giamarchi, T. Esslinger, and J.-P. Brantut, Connecting Strongly Correlated Superfluids by a Quantum Point Contact, Science 350, 1498 (2015).

[42] N. R. Cooper, Rapidly Rotating Atomic Gases, Adv. Phys. 57, 539 (2008).

[43] N. Goldman, J. C. Budich, and P. Zoller, Topological Quantum Matter with Ultracold Gases in Optical Lattices, Nat. Phys. 12, 639 (2016).

[44] N. R. Cooper, J. Dalibard, and I. B. Spielman, Topological Bands for Ultracold Atoms, arXiv:1803.00249.

[45] J. Dalibard, F. Gerbier, G. Juzeliūnas, and P. Öhberg, Colloquium: Artificial Gauge Potentials for Neutral Atoms, Rev. Mod. Phys. 83, 1523 (2011).

[46] N. Goldman, G. Juzeliūnas, P. Öhberg, and I. B. Spielman, Light-Induced Gauge Fields for Ultracold Atoms, Rep. Prog. Phys. 77, 126401 (2014).

[47] O. Boada, A. Celi, J. I. Latorre, and M. Lewenstein, Quantum Simulation of an Extra Dimension, Phys. Rev. Lett. 108, 133001 (2012).

[48] A. Celi, P. Massignan, J. Ruseckas, N. Goldman, I. B. Spielman, G. Juzeliūnas, and M. Lewenstein, Synthetic Gauge Fields in Synthetic Dimensions, Phys. Rev. Lett. 112, 043001 (2014).

[49] N. R. Cooper and A. M. Rey, Adiabatic Control of Atomic Dressed States for Transport and Sensing, Phys. Rev. A 92, 021401(R) (2015).

[50] B. Gadway, Atom-Optics Approach to Studying Transport Phenomena, Phys. Rev. A 92, 043606 (2015).

[51] M. Mancini, G. Pagano, G. Cappellini, L. Livi, M. Rider, J. Catani, C. Sias, P. Zoller, M. Inguscio, M. Dalmonte, and L. Fallani, Observation of Chiral Edge States with Neutral Fermions in Synthetic Hall Ribbons., Science 349, 1510 (2015).

[52] B. K. Stuhl, H.-I. Lu, L. M. Aycock, D. Genkina, and I. B. Spielman, Visualizing Edge States with an Atomic Bose Gas in the Quantum Hall Regime, Science 349, 1514 (2015).

[53] E. J. Meier, F. A. An, and B. Gadway, Atom-Optics Simulator of Lattice Transport Phenomena, Phys. Rev. A 93, 051602(R) (2016).

[54] L. F. Livi, G. Cappellini, M. Diem, L. Franchi, C. Clivati, M. Frittelli, F. Levi, D. Calonico, J. Catani, M. Inguscio, and L. Fallani, Synthetic Dimensions and Spin-Orbit Coupling with an Optical Clock Transition, Phys. Rev. Lett. 117, 220401 (2016).

[55] F. A. An, E. J. Meier, and B. Gadway, Direct Observation of Chiral Currents and Magnetic Reflection in Atomic Flux Lattices, Sci. Adv. 3, e1602685 (2017).

[56] H. M. Price, T. Ozawa, and N. Goldman, Synthetic Dimensions for Cold Atoms from Shaking a Harmonic Trap, Phys. Rev. A 95, 023607 (2017).

[57] S. Kolkowitz, S. L. Bromley, T. Bothwell, M. L. Wall, G. E. Marti, A. P. Koller, X. Zhang, A. M. Rey, and J. Ye, 
Spin-Orbit-Coupled Fermions in an Optical Lattice Clock, Nature (London) 542, 66 (2017).

[58] B. Bauer, T. Pereg-Barnea, T. Karzig, M.-T. Rieder, G. Refael, E. Berg, and Y. Oreg, Topologically Protected Braiding in a Single Wire Using Floquet Majorana Modes, Phys. Rev. B 100, 041102 (2019).

[59] T. Ozawa, H. M. Price, N. Goldman, O. Zilberberg, and I. Carusotto, Synthetic Dimensions in Integrated Photonics: From Optical Isolation to Four-Dimensional Quantum Hall Physics, Phys. Rev. A 93, 043827 (2016).

[60] L. Yuan, Q. Lin, M. Xiao, and S. Fan, Synthetic Dimension in Photonics, Optica 5, 1396 (2018).

[61] E. Lustig, S. Weimann, Y. Plotnik, Y. Lumer, M. A. Bandres, A. Szameit, and M. Segev, Photonic Topological Insulator in Synthetic Dimensions, arXiv:1807.01983.

[62] Q. Lin, X.-Q. Sun, M. Xiao, S.-C. Zhang, and S. Fan, Constructing Three-Dimensional Photonic Topological Insulator Using Two-Dimensional Ring Resonator Lattice with a Synthetic Frequency Dimension, arXiv:1802.02597.

[63] M. Lebrat, P. Grišins, D. Husmann, S. Häusler, L. Corman, T. Giamarchi, J.-P. Brantut, and T. Esslinger, Band and Correlated Insulators of Cold Fermions in a Mesoscopic Lattice, Phys. Rev. X 8, 011053 (2018).

[64] A. R. Kolovsky, Creating Artificial Magnetic Fields for Cold Atoms by Photon-Assisted Tunneling, Europhys. Lett. 93, 20003 (2011).

[65] C. E. Creffield and F. Sols, Comment on "Creating Artificial Magnetic Fields for Cold Atoms by PhotonAssisted Tunneling” by Kolovsky A. R., Europhys. Lett. 101, 40001 (2013).

[66] A. Bermudez, T. Schaetz, and D. Porras, Synthetic Gauge Fields for Vibrational Excitations of Trapped Ions, Phys. Rev. Lett. 107, 150501 (2011).

[67] N. Goldman, J. Dalibard, M. Aidelsburger, and N. R. Cooper, Periodically Driven Quantum Matter: The Case of Resonant Modulations, Phys. Rev. A 91, 033632 (2015).

[68] C. E. Creffield, G. Pieplow, F. Sols, and N. Goldman, Realization of Uniform Synthetic Magnetic Fields by Periodically Shaking an Optical Square Lattice, New J. Phys. 18, 093013 (2016).

[69] T. Kitagawa, E. Berg, M. Rudner, and E. Demler, Topological Characterization of Periodically Driven Quantum Systems, Phys. Rev. B 82, 235114 (2010).

[70] N. Goldman and J. Dalibard, Periodically Driven Quantum Systems: Effective Hamiltonians and Engineered Gauge Fields, Phys. Rev. X 4, 031027 (2014).

[71] M. Bukov, L. D’Alessio, and A. Polkovnikov, Universal High-Frequency Behavior of Periodically Driven Systems: From Dynamical Stabilization to Floquet Engineering, Adv. Phys. 64, 139 (2015).

[72] A. Eckardt, Colloquium: Atomic Quantum Gases in Periodically Driven Optical Lattices, Rev. Mod. Phys. 89, 011004 (2017).

[73] S. Kohler, J. Lehmann, and P. Hänggi, Driven Quantum Transport on the Nanoscale, Phys. Rep. 406, 379 (2005).

[74] T. Kitagawa, T. Oka, A. Brataas, L. Fu, and E. Demler, Transport Properties of Nonequilibrium Systems under the Application of Light: Photoinduced Quantum Hall Insulators without Landau Levels, Phys. Rev. B 84, 235108 (2011).
[75] H. H. Yap, L. Zhou, J.-S. Wang, and J. Gong, Computational Study of the Two-Terminal Transport of Floquet Quantum Hall Insulators, Phys. Rev. B 96, 165443 (2017).

[76] D. R. Hofstadter, Energy Levels and Wave Functions of Bloch Electrons in Rational and Irrational Magnetic Fields, Phys. Rev. B 14, 2239 (1976).

[77] N. Tsuji, T. Oka, and H. Aoki, Correlated Electron Systems Periodically Driven out of Equilibrium: Floquet + DMFT Formalism, Phys. Rev. B 78, 235124 (2008).

[78] F. Gagel and K. Maschke, Finite-Difference Approach to Edge-State Transport in Quantum Wires and Multiterminal Devices, Phys. Rev. B 52, 2013 (1995).

[79] F. Gagel and K. Maschke, Potential Barriers in Quantum Hall Devices, Phys. Rev. B 54, 10346 (1996).

[80] D. A. Ryndyk, R. Gutiérrez, B. Song, and G. Cuniberti, Green Function Techniques in the Treatment of Quantum Transport at the Molecular Scale (Springer, Berlin, 2009), Vol. 93.

[81] C. H. Lewenkopf and E. R. Mucciolo, The Recursive Green's Function Method for Graphene, J. Comput. Electron. 12, 203 (2013).

[82] C. Caroli, R. Combescot, P. Nozieres, and D. Saint-James, Direct Calculation of the Tunneling Current, J. Phys. C 4, 916 (1971).

[83] D. J. Thouless and S. Kirkpatrick, Conductivity of the Disordered Linear Chain, J. Phys. C 14, 235 (1981).

[84] G. Thorgilsson, G. Viktorsson, and S. I. Erlingsson, Recursive Green's Function Method for Multi-Terminal Nanostructures, J. Comput. Phys. 261, 256 (2014).

[85] M. Büttiker, Four-Terminal Phase-Coherent Conductance, Phys. Rev. Lett. 57, 1761 (1986).

[86] M. Büttiker, Absence of Backscattering in the Quantum Hall Effect in Multiprobe Conductors, Phys. Rev. B 38, 9375 (1988).

[87] C. L. Kane, R. Mukhopadhyay, and T. C. Lubensky, Fractional Quantum Hall Effect in an Array of Quantum Wires, Phys. Rev. Lett. 88, 036401 (2002).

[88] J. C. Budich, A. Elben, M. Łącki, A. Sterdyniak, M. A. Baranov, and P. Zoller, Coupled Atomic Wires in a Synthetic Magnetic Field, Phys. Rev. A 95, 043632 (2017).

[89] M. Moskalets and M. Büttiker, Floquet Scattering Theory of Quantum Pumps, Phys. Rev. B 66, 205320 (2002).

[90] A. Eckardt and E. Anisimovas, High-Frequency Approximation for Periodically Driven Quantum Systems from a Floquet-Space Perspective, New J. Phys. 17, 093039 (2015).

[91] A. Farrell and T. Pereg-Barnea, Photon-Inhibited Topological Transport in Quantum Well Heterostructures, Phys. Rev. Lett. 115, 106403 (2015).

[92] J.-P. Brantut, C. Grenier, J. Meineke, D. Stadler, S. Krinner, C. Kollath, T. Esslinger, and A. Georges, A Thermoelectric Heat Engine with Ultracold Atoms, Science 342, 713 (2013).

[93] D. Husmann, M. Lebrat, S. Häusler, J.-P. Brantut, L. Corman, and T. Esslinger, Breakdown of the WiedemannFranz Law in a Unitary Fermi Gas, Proc. Natl. Acad. Sci. U.S.A. 115, 8563 (2018).

[94] M. Schmidt, S. Kessler, V. Peano, O. Painter, and F. Marquardt, Optomechanical Creation of Magnetic Fields for Photons on a Lattice, Optica 2, 635 (2015). 
[95] L. Yuan, Y. Shi, and S. Fan, Photonic Gauge Potential in a System with a Synthetic Frequency Fimension, Opt. Lett. 41, 741 (2016).

[96] T. Ozawa and I. Carusotto, Synthetic Dimensions with Magnetic Fields and Local Interactions in Photonic Lattices, Phys. Rev. Lett. 118, 013601 (2017).

[97] C.-H. Wang and J. M. Taylor, Landauer Formulation of Photon Transport in Driven Systems, Phys. Rev. B 94, 155437 (2016).

[98] T. Ozawa, H. M. Price, A. Amo, N. Goldman, M. Hafezi, L. Lu, M. Rechtsman, D. Schuster, J. Simon, O. Zilberberg, and I. Carusotto, Topological Photonics, Rev. Mod. Phys. 91, 015006 (2019).

[99] C. W. Groth, M. Wimmer, A. R. Akhmerov, and X. Waintal, KWANT: A Software Package for Quantum Transport, New J. Phys. 16, 063065 (2014).

[100] H. Bruus and K. Flensberg, Many-Body Quantum Theory in Condensed Matter Physics: An Introduction (Oxford University Press, New York, 2004).

[101] Y. Meir and N. S. Wingreen, Landauer Formula for the Current through an Interacting Electron Region, Phys. Rev. Lett. 68, 2512 (1992).
[102] H. Haug and A.-P. Hauho, Quantum Kinetics and Optics of Semiconductors (Springer-Verlag, Berlin, 2008).

[103] A. Eckardt, C. Weiss, and M. Holthaus, SuperfluidInsulator Transition in a Periodically Driven Optical Lattice, Phys. Rev. Lett. 95, 260404 (2005).

[104] H. Lignier, C. Sias, D. Ciampini, Y. Singh, A. Zenesini, O. Morsch, and E. Arimondo, Dynamical Control of Matter-Wave Tunneling in Periodic Potentials, Phys. Rev. Lett. 99, 220403 (2007).

[105] E. Kierig, U. Schnorrberger, A. Schietinger, J. Tomkovic, and M.K. Oberthaler, Single-Particle Tunneling in Strongly Driven Double-Well Potentials, Phys. Rev. Lett. 100, 190405 (2008).

[106] J. Struck, C. Ölschläger, M. Weinberg, P. Hauke, J. Simonet, A. Eckardt, M. Lewenstein, K. Sengstock, and P. Windpassinger, Tunable Gauge Potential for Neutral and Spinless Particles in Driven Optical Lattices, Phys. Rev. Lett. 108, 225304 (2012).

[107] I. Esin, M. S. Rudner, G. Refael, and N. H. Lindner, Quantized Transport and Steady States of Floquet Topological Insulators, Phys. Rev. B 97, 245401 (2018). 